\title{
On the Euler-Kronecker constants of global fields and primes with small norms
}

\author{
Yasutaka Ihara
}

Dedicated to V.Drinfeld

\section{Introduction}

Let $K$ be a global field, i.e., either an algebraic number field of finite degree (abbrev. $\mathrm{NF}$ ), or an algebraic function field of one variable over a finite field $(\mathrm{FF})$. Let $\zeta_{K}(s)$ be the Dedekind zeta function of $K$, with the Laurent expansion at $s=1$ :

$$
\zeta_{K}(s)=c_{-1}(s-1)^{-1}+c_{0}+c_{1}(s-1)+\cdots \quad\left(c_{-1} \neq 0\right) .
$$

In this paper, we shall present a systematic study of the real number

$$
\gamma_{K}=c_{0} / c_{-1}
$$

attached to each $K$, which we call the Euler-Kronecker constant (or invariant) of $K$. When $K=\mathbb{Q}$ (the rational number field), it is nothing but the Euler-Mascheroni constant

$$
\gamma_{\mathbb{Q}}=\lim _{n \rightarrow \infty}\left(1+\frac{1}{2}+\cdots+\frac{1}{n}-\log n\right)=0.57721566 \cdots,
$$

and when $K$ is imaginary quadratic, the well-known Kronecker limit formula expresses $\gamma_{K}$ in terms of special values of the Dedekind $\eta$ function. This constant $\gamma_{K}$ appears here and there in several articles in analytic number theory, but as far as the author knows, it has not played a main role nor has it been studied so systematically. We shall consider $\gamma_{K}$ more as an invariant of $K$.

Before explaining our motivation for systematic study, let us briefly look at the FFcase. When $K$ is the function field of a curve $X$ over a finite field $\mathbb{F}_{q}$ with genus $g$, so that $\zeta_{K}(s)$ is a rational function of $u=q^{-s}$ of the form

$$
\zeta_{K}(s)=\frac{\prod_{\nu=1}^{g}\left(1-\pi_{\nu} u\right)\left(1-\bar{\pi}_{\nu} u\right)}{(1-u)(1-q u)}, \quad \pi_{\nu} \bar{\pi}_{\nu}=q \quad(1 \leq \nu \leq g),
$$

then $\gamma_{K}$ is closely related to the harmonic mean of $g$ positive real numbers

$$
\left(1-\pi_{\nu}\right)\left(1-\bar{\pi}_{\nu}\right) \quad(1 \leq \nu \leq g),
$$


in contrast to the facts that their arithmetic (resp. geometric) means are related to the number of $\mathbb{F}_{q}$-rational points of $X$ (resp. its Jacobian $J_{X}$ ). More explicitly,

$$
\begin{aligned}
\frac{\gamma_{K}}{\log q} & =(q-1) \sum_{\nu=1}^{g} \frac{1}{\left(1-\pi_{\nu}\right)\left(1-\bar{\pi}_{\nu}\right)}-(g-1)-\frac{q+1}{2(q-1)} \\
& =\sum_{m=1}^{\infty}\left(\frac{q^{m}+1-N_{m}}{q^{m}}\right)+1-\frac{q+1}{2(q-1)}
\end{aligned}
$$

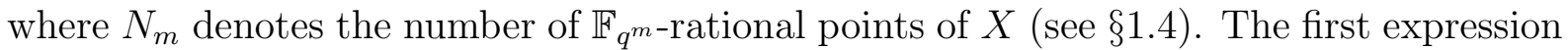
shows that $\gamma_{K}$ is a rational multiple of $\log q$, while the second shows that when $X$ has

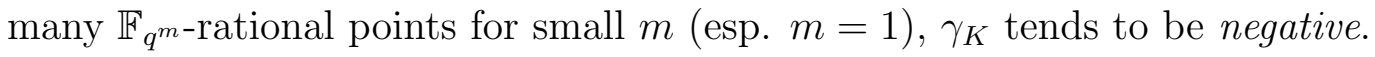

Our first basic observation is that, including the NF-case, $\gamma_{K}$ can sometimes be "conspicuously negative", and that this occurs when $K$ has "many primes with small norms". In the FF-case, there are known interesting towers of curves over $\mathbb{F}_{q}$ with many rational points, and we ask how negative $\gamma_{K}$ can be, in general and for such a tower. In the NF-case, there is no notion of rational points, but those $K$ having many primes with small norms would be equally interesting for applications (to coding theory, etc.). Moreover, the related problems often have their own arithmetic significance (e.g. the fields $K_{p}$ described below). We wish to know how negative $\gamma_{K}$ can be also in the NF-case. A careful comparison of the two cases is very interesting. Thus we are led to studying $\gamma_{K}$ in both cases under a unified treatment, basically assuming the generalized Riemann hypothesis (GRH) in the NF-case. We shall give a method for systematic computation of $\gamma_{K}$, give some general upper and lower bounds, and study three special cases more closely, including that of curves with many rational points, for comparisons and applications.

In Part 1, after basic preliminaries, we shall give some explicit estimations of $\gamma_{K}$, and also discuss possibilities of improvements when we specialize to smaller families of $K$ (see $\S 1.6)$. Among them, Theorem 1 gives a general upper bound for $\gamma_{K}$. The main term of this upper bound is

$d=d_{K}$ being the discriminant. The lower bound is, as we shall see, necessarily much weaker. First, the main term of our general lower bound (Proposition 3) reads as

$$
\begin{cases}-\log \sqrt{|d|} & (N F, \text { unconditionally }) \\ -(g-1) \log q & (F F) .\end{cases}
$$

Secondly, when we fix $q$, the latter will be improved to be

$$
-\frac{1}{\sqrt{q}+1}(g-1) \log q
$$


(Theorem 2). In other words,

$$
C(q):=\liminf \frac{\gamma_{K}}{\left(g_{K}-1\right) \log q} \geq-\frac{1}{\sqrt{q}+1} .
$$

This is based on a result of Tsfasman $\left[\mathrm{Ts}_{1}\right]$, and is somewhat stronger than what we can prove only by using the Drinfeld-Vladut asymptotic bound [D-V] for $N_{1}$. We shall moreover see that the equality holds in (0.9) when $q$ is a square (see below). In the NF-case, our attention will be focused on the absolute constant

$$
C=\liminf \frac{\gamma_{K}}{\log \sqrt{\left|d_{K}\right|}} .
$$

Clearly, (0.7) gives $C \geq-1$ (unconditionally), but quite recently, Tsfasman proved, as a beautiful application of $[\mathrm{T}-\mathrm{V}]$, that

$$
C \geq-0.26049 \ldots \quad \text { (under } G R H \text { ) }
$$

( $\left[\mathrm{Ts}_{2}\right]$ in this Volume). The estimation of $C(q)$ or $C$ from above is related to finding a sequence of $K$ having many primes with small norms. As for $C(q)$, see below. As for $C$, the author obtained $C \leq-0.1635$ (under $\mathrm{GRH}$; see $\S 1.6$ ), but $\left[\mathrm{Ts}_{2}\right]$ contains a sharper unconditional estimation. At any rate, in each of the FF- and the NF-case, we see that the general (negative) lower bound for $\gamma_{K}$ cannot be so close to 0 as the (positive) upper bound.

Thirdly, when the degree $N$ of $K$ over $\mathbb{Q}$ resp. $\mathbb{F}_{q}(t)$ is fixed $(N>1)$, or grows slowly enough, (0.7) will be improved to be

$$
\begin{cases}-2(N-1) \log \left(\frac{\log \sqrt{|d|}}{(N-1)}\right) & (N F, \text { under } G R H), \\ -2(N-1) \log \left(\frac{(g-1) \log q}{N-1}\right) & (F F)\end{cases}
$$

(Theorem 3), which is nearly as strong as the upper bound, and exactly so (with opposite signs) when $N=2$. Granville-Stark [G-S] (§3.1) gave an equivalent statement when $N=2$ (NF-case), and our Theorem 3 was inspired by this work. The bound (0.12) is quite sharp. In fact, some families of $K$ having many primes with small norms insist that (0.12) cannot be replaced by its quotient even by $\log \log N$. To be precise, it cannot be replaced by its quotient by any such $f(N)(\mathrm{NF})$ (resp. $f_{q}(N)$ for a fixed $q>2(\mathrm{FF})$ ) as satisfying $f(N) \rightarrow \infty$ (resp. $f_{q}(N) \rightarrow \infty$ ).

$\S 1.7$ is for supplementary remarks related to computations of $\gamma_{K}$.

In Part 2, we shall study some special cases. First, let $q$ be any fixed prime power. Then, as an application of a result in [E-], we obtain

$$
C(q) \leq-c_{0} \frac{\log q}{q-1}
$$


$(\S 2.1)$, where $c_{0}$ is a certain positive absolute constant. Then we treat the case where $K$ is the function field over $\mathbb{F}_{q}$ of a Shimura curve, with $q$ a square, and $g_{K} \gg q(\S 2.1)$. In this case, as a reflection of the fact that such a curve has so many $\mathbb{F}_{q}$-rational points, we can prove

$$
\gamma_{K} \leq-\frac{1}{\sqrt{q}+1}\left(g_{K}-1\right) \log q+\varepsilon
$$

Therefore, combining this with (0.9), we obtain

$$
C(q)=-\frac{1}{\sqrt{q}+1} \quad(q: \text { a square }) .
$$

Secondly, when $K$ is imaginary quadratic, we combine our upper bound for $\gamma_{K}$ with the Kronecker limit formula, to give a lower bound for its class number $h_{K}$;

$$
\frac{h_{K} \log \left|d_{K}\right|}{\sqrt{\left|d_{K}\right|}}>\frac{\pi}{3}-\varepsilon
$$

with an explicit description of the $\varepsilon$-part (under GRH)(Theorem 5 §2.2). As asymptotic formula, this is weaker than Littlewood's [Li] and almost equivalent with Granville-Stark's [G-S] (both conditional) formulas; its merit is explicitness.

Thirdly, we consider the case where $K=K_{p}$ is the "first layer" of the cyclotomic $\mathbb{Z}_{p}$-extension over $\mathbb{Q}(\S 2.3)$. It is the unique cyclic extension over $\mathbb{Q}$ of degree $p$ contained in the field of $p^{2}$-th roots of unity. By classfield theory, a prime $\ell$ decomposes completely in $K_{p}$ if and only if

$$
\ell^{p-1} \equiv 1 \quad\left(\bmod p^{2}\right)
$$

We shall apply our estimations of $\gamma_{K}$ to this case $K=K_{p}$ (Theorem 6 and its Corollaries). Among them, Corollary 1 gives information on small $\ell$ 's satisfying (0.17) for a fixed large $p$, while Corollary 3 relates the question on the existence of "many" $p$ satisfying $(0.17)$ for a fixed $\ell$ to that on $\lim \inf \left(\gamma_{K_{p}} / p\right)$. (Incidentally, $\lim \left(\gamma_{K_{p}} / \log \sqrt{d_{K_{p}}}\right)=\lim \left(\gamma_{K_{p}} /(p-\right.$ 1) $\log p)=0$ under GRH.) From Table 2.3A, see how the existence of a very small $\ell$ satisfying (0.17) pushes the value of $\gamma_{K_{p}}$ drastically towards left on the negative real axis. For example, (0.17) is satisfied for $\ell=2$ and $p=1093$, and accordingly, $\gamma_{K_{1093}}$ is so negative as about -747 , while for several neighboring primes $p$, the absolute values of $\gamma_{K_{p}}$ are at most 10 . Finally in $\S 2.4 \sim 2.5$, we shall give some application to the "field index" of $K_{p}$.

Our main tool is "the explicit formula" for the prime counting function

$$
\Phi_{K}(x)=\frac{1}{x-1} \sum_{N(P)^{k} \leq x}\left(\frac{x}{N(P)^{k}}-1\right) \log N(P) \quad(x>1),
$$


where $(P, k)$ runs over the pairs of (non-archimedean) primes $P$ of $K$ and positive integers $k$ such that $N(P)^{k} \leq x(\S 2.2 \sim 2.3)$. This function $\Phi_{K}(x)$ is quite close to $\log x$ when $x$ is large, and the connection with our constant $\gamma_{K}$ is

$$
\begin{array}{ll}
\lim _{x \rightarrow \infty}\left(\log x-\Phi_{K}(x)\right)=\gamma_{K}+1 & (N F, \text { unconditionally), } \\
\lim _{\substack{x \in q^{\mathbb{Z}} \\
x \rightarrow \infty}}\left(\log x-\Phi_{K}(x)\right)=\gamma_{K}+\frac{q+1}{2(q-1)} \log q & (F F) .
\end{array}
$$

It is a simple combination of two well-known prime counting functions, but two characteristic features of $\Phi_{K}(x)$ are : (i) it is continuous, and (ii) the oscillating term in the explicit formula for $\Phi_{K}(x)$ has the form

$$
-\frac{1}{2(x-1)} \lim _{T \rightarrow \infty} \sum_{|\rho|<T} \frac{\left(x^{\rho}-1\right)\left(x^{1-\rho}-1\right)}{\rho(1-\rho)},
$$

where $\rho$ runs over the non-trivial zeros of $\zeta_{K}(s)$, which, under GRH, is very easy to evaluate. In fact, then it is sandwiched in-between two multiples, by $((\sqrt{x}+1) /(\sqrt{x}-1))^{ \pm 1}$, of the negative real constant

$$
-\frac{1}{2} \sum_{\rho} \frac{1}{\rho(1-\rho)}
$$

And $-\gamma_{K}$ is a translate of $(0.22)$ by a more elementary constant associated to $K$. This is why (under GRH in the NF-case) we can obtain results always with explicit error terms, and only by simple elementary arguments. Usually, one uses the "truncated explicit formula" where the summation over $\rho$ is restricted to $|\rho|<T$ and instead contains an error term $R(x, T)$ which is not easy to evaluate systematically.

We add here three more observations.

(i) In some sense, the quantity on the RHS of (0.19)(0.20) may be more canonical than $\gamma_{K}$ as an invariant of $K$. Note that (0.20) with $q=1$ "corresponds to" (0.19), and that (0.5) will be simplified if we use the RHS of (0.20) instead of $\gamma_{K}$ itself (see $\S 1.4$ ).

(ii) One can of course generalize the definition of $\gamma_{K}$ to the case of $L$-functions, although then they will not usually be real numbers. Multiplicative relations among the $L$-functions give rise to additive relations among these constants. In particular, when $H$ runs over the subgroups of a given finite group $G$, any linear relation among those characters of $G$ induced from the trivial character of $H$ gives rise to the corresponding linear relation among the $\gamma_{K}$, where $K$ runs over the intermediate extensions of a given G-extension.

(iii) When $K$ is either the cyclotomic field $\mathbb{Q}\left(\boldsymbol{\mu}_{m}\right)$ or its maximal real subfield $\mathbb{Q}\left(\boldsymbol{\mu}_{m}\right)^{+}$, it seems fairly likely that $\gamma_{K}$ is always positive!. The author has computed $\gamma_{K}$ in both cases 
up to $m=600$, and Mahoro Shimura more recently checked the first case $K=\mathbb{Q}\left(\boldsymbol{\mu}_{m}\right)$ for $m$ as far as up to 8000, and we have found no counterexamples. On the other hand, their difference, "the relative" $\gamma_{K}$, seems to take both signs "almost equally".

Studies of $\gamma_{K}$ for various families of global fields $K$ including these cases will be left to future publications. Some open problems and numerical data can be found in my article in the (informal) "Proceedings of the 2004 Workshop on Cryptography and Related Mathematics", Chuo University. The 2003-Worshop Proceedings contains a short summary of the present paper.

\section{Part 1 The "explicit formula" for $\Phi_{K}(x)$, and estimations of $\gamma_{K}$}

\subsection{The function $\Phi_{K}(x)$}

Let $K$ be a global field. We denote by $P$ any (non-archimedean) prime divisor of $K$, and by $N(P)$ its norm. As mentioned in the Introduction, we shall consider the prime counting function

$$
\Phi_{K}(x)=\frac{1}{x-1} \sum_{N(P)^{k} \leq x}\left(\frac{x}{N(P)^{k}}-1\right) \log N(P) \quad(x>1) .
$$

Here, $(P, k)$ runs over all pairs with $k \geq 1$ and $N(P)^{k} \leq x$ (or what amounts to the same effect, $\left.N(P)^{k}<x\right)$. Call a point on the real axis critical if it is of the form $N(P)^{k}$. Then $\Phi_{K}(x)$ remains to be 0 until the first critical point, then monotone increasing, and is everywhere continuous. In fact, at each critical point $\Phi_{K}(x)$ acquires new summands but their values are 0 at this point, so the visible increase at each critical point is that of the slope. The slope of $\Phi_{K}(x)$ between two adjacent critical points $a<b$ is $c(x-1)^{-2}$, where

$$
c=\sum_{N(P)^{k} \leq a}\left(1-\frac{1}{N(P)^{k}}\right) \log N(P)>0 .
$$

So, the slope near $x$ is close to $\left(\sum_{N(P)^{k}<x} \log N(P)\right) x^{-2} \sim x^{-1}$. Thus, $\Phi_{K}(x)$ is an arithmetic approximation of $\log x$. If the field $K$ has many primes $P$ with small $N(P)$, then $\Phi_{K}(x)$ increases faster than $\log x$, at least for some while. The difference $\log x-\Phi_{K}(x)$ "at infinity" is closely related to $\gamma_{K}$, as we shall see later. 


\subsection{The explicit formula for $\Phi_{K}(x)$}

From Weil's general explicit formula $\left[\mathrm{W}_{1}\right],\left[\mathrm{W}_{2}\right]$, we obtain, as will be indicated in $\S 1.3$, the following formula for $\Phi_{K}(x)$;

$$
\Phi_{K}(x)=\log x+\left(\alpha_{K}+\beta_{K}\right)+\ell_{K}(x)+r_{K}(x) \quad(x>1) .
$$

Here,

$$
\begin{aligned}
\alpha_{K} & =\frac{1}{2} \log |d| & & (N F) \\
& =(g-1) \log q & & (F F),
\end{aligned}
$$

( $d=d_{K}$ : the discriminant, $g=g_{K}$ : the genus, $\mathbb{F}_{q}$ : the exact constant field),

$$
\begin{array}{rlr}
\beta_{K} & =-\left\{\frac{r_{1}}{2}(\gamma+\log 4 \pi)+r_{2}(\gamma+\log 2 \pi)\right\} & (N F) \\
& =0 & (F F),
\end{array}
$$

$\left(r_{1}, r_{2}\right.$ : the number of real, imaginary places of $K$, respectively, $\gamma=\gamma_{\mathbb{Q}}$ : the EulerMascheroni constant $=0.57721566 \cdots)$,

$$
\begin{array}{rlr}
\ell_{K}(x) & =\frac{r_{1}}{2}\left(\log \frac{x+1}{x-1}+\frac{2}{x-1} \log \frac{x+1}{2}\right)+r_{2}\left(\log \frac{x}{x-1}+\frac{1}{x-1} \log x\right) & (N F) \\
& =\phi(q, x) & (F F)
\end{array}
$$

where $\phi(q, x)$ is a certain continuous function of $x$ parametrized by $q$, satisfying

$$
\begin{aligned}
0 \leq \phi(q, x) & <\log q \\
\phi(q, x) & =0 \longleftrightarrow x=q^{m} \text { with some } m \in \mathbb{N}
\end{aligned}
$$

(see below). Finally,

$$
r_{K}(x)=-\frac{1}{2(x-1)} \sum_{\rho} \frac{\left(x^{\rho}-1\right)\left(x^{1-\rho}-1\right)}{\rho(1-\rho)},
$$

where $\rho$ runs over all non-trivial zeros of $\zeta_{K}(s)$, counted with multiplicities, and

$$
\sum_{\rho}=\lim _{T \rightarrow \infty} \sum_{|\rho|<T}
$$

By the functional equation for $\zeta_{K}(s)$, if $\rho$ is a non-trivial zero of $\zeta_{K}(s)$ then so is $1-\rho$, with the same multiplicity. 
In the FF-case, when $x=q^{m}(m \in \mathbb{N})$,

$$
\left\{\begin{array}{l}
\Phi_{K}(x) / \log q=\frac{1}{q^{m}-1} \sum_{k \operatorname{deg} P \leq m}\left(q^{m-k \operatorname{deg} P}-1\right) \operatorname{deg} P \\
\log x / \log q \\
\alpha_{K} / \log q \\
\beta_{K} / \log q \\
r_{K}(x) / \log q=-1, \\
=\ell_{K}(x) / \log q=0,
\end{array}\right.
$$

where

$$
\zeta_{K}(s)=\frac{\prod_{\nu=1}^{g}\left(1-\pi_{\nu} u\right)\left(1-\bar{\pi}_{\nu} u\right)}{(1-u)(1-q u)}, \quad u=q^{-s}, \quad \pi_{\nu} \bar{\pi}_{\nu}=q \quad(1 \leq \nu \leq g) .
$$

(To derive the last formula for $r_{K}\left(q^{m}\right) / \log q$ from the definition (1.2.6) of $r_{K}(x)$, take any $\alpha \in \mathbb{C}^{\times}$ and $q>1$, and substitute $e^{z}=\alpha^{-1} q^{s}$ in the partial fraction expansion formula

$$
\left(e^{z}-1\right)^{-1}+1 / 2=\lim _{T \rightarrow \infty} \sum_{n=-T}^{T}(z-2 \pi i n)^{-1},
$$

which gives

$$
\frac{\log q}{\alpha^{-1} q^{s}-1}+\frac{\log q}{2}=\lim _{T \rightarrow \infty} \sum_{\substack{q^{\rho}=\alpha \\|\rho| \leq T}}(s-\rho)^{-1} .
$$

Now let $q=\alpha \bar{\alpha}, s=0$ and take the real part of (1.2.11) to obtain

$$
\frac{q-1}{(\alpha-1)(\bar{\alpha}-1)} \log q=\lim _{T \rightarrow \infty} \sum_{\substack{q^{\rho}=\alpha \\|\rho| \leq T}}\left(\frac{1}{\rho}+\frac{1}{\bar{\rho}}\right) .
$$

The desired formula follows immediately from this.)

Note that each reciprocal zero $\pi_{\nu}$ (resp. $\bar{\pi}_{\nu}$ ) of $\zeta_{K}(s)$ in $u=q^{-s}$ corresponds to infinitely many zeros in $s$, which are translations of one of them by $2 \pi i n / \log q(n \in \mathbb{Z})$. It also has poles at all translations of 0,1 by $2 \pi i n / \log q(n \in \mathbb{Z})$. The function $\phi(q, x)$ arises from the poles $\theta \neq 0,1$;

$$
\phi(q, x)=\frac{1}{2(x-1)} \sum_{\text {poles } \theta \neq 0,1} \frac{\left(x^{\theta}-1\right)\left(x^{1-\theta}-1\right)}{\theta(1-\theta)},
$$

where

$$
\sum_{\theta}=\lim _{T \rightarrow \infty} \sum_{|\theta|<T}
$$


Since either $q^{\theta}=1$ or $q^{1-\theta}=1$, it is clear that $\phi(q, x)=0$ when $x=q^{m}(m \in \mathbb{N})$. In a finite form,

$$
\phi(q, x)=\log \left(\frac{q^{m}}{x}\right)-\frac{\left(q^{m-1}-1\right)\left(q^{m}-x\right)}{(x-1)\left(q^{m}-q^{m-1}\right)} \log q
$$

for $q^{m-1} \leq x \leq q^{m} \quad(m \in \mathbb{Z}, m \geq 1, x \neq 1)$. This follows immediately from the following

Proposition 1 (i). The functions $\ell_{K}(x)$ and $r_{K}(x)$ are continuous.

(ii). (FF) $(x-1)\left(\ell_{K}(x)+\log x\right)$ and $(x-1) r_{K}(x)$ are linear on each interval $q^{m-1} \leq$ $x \leq q^{m}(m \geq 1)$.

Proof $(\mathrm{NF}) \ell_{K}(x)$ is continuous by definition. Since $\Phi_{K}(x)$ and $\ell_{K}(x)$ are both continuous, $r_{K}(x)$ is also continuous by (1.2.1).

(FF) In this case, $\ell_{K}(x)=\phi(q, x)$ is a function of $x$ determined only by $q$. By (1.2.1) applied to the case $g=0$, we have

$$
\phi(q, x)=\Phi_{\mathbb{F}_{q}(t)}(x)-\log x+\log q ;
$$

hence $\phi(q, x)$ is continuous. Now, when $q^{m-1} \leq x \leq q^{m}$,

$$
(x-1) \Phi_{K}(x)=\sum_{N(P)^{k} \leq q^{m-1}}\left(\frac{x}{N(P)^{k}}-1\right) \log N(P)
$$

is linear. Hence by $(1.2 .16),(x-1)(\phi(q, x)+\log x)$ is also linear on this interval. Moreover, the function

$$
(x-1) r_{K}(x)=(x-1) \Phi_{K}(x)-(x-1)(\phi(q, x)+\log x)-(x-1)\left(\alpha_{K}+\beta_{K}\right)
$$

is also linear in the same interval.

Remarks (i) In the NF-case, $\beta_{K}$ and $\ell_{K}(x)$ both come from the archimedean places. Among them, $\beta_{K}$ is the value at $s=1$ of the logarithmic derivative of the "standard $\Gamma$ factor" of $\zeta_{K}(s)$ (see $\S 1.3$ below), and $\ell_{K}(x)$ comes from the trivial zeros of $\zeta_{K}(s)$. Thus, $\ell_{K}(s)$ for the $(\mathrm{FF})$ and the $(\mathrm{NF})$ cases have quite different origins $\cdots$ poles $\neq 0,1$, vs. trivial zeros. We have given them the same name here only to save notation.

(ii) In the NF-case, $\beta_{K}+\ell_{K}(x)$ is the term coming from the archimedean places, and our separation into $\beta_{K}$ and $\ell_{K}(x)$ can also be characterized by

$$
\lim _{x \rightarrow \infty} \ell_{K}(x)=0
$$

(cf. Lemma 2 below $(\S 1.5)$ ).

(iii) We note also that

$$
\ell_{K}(x) \geq 0 \quad(x>1)
$$

in both cases (cf. Lemma 2, §1.5). 


\subsection{The explicit formula for $\Phi_{K}(x)$ (continued)}

The above explicit formula (1.2.1) for $\Phi_{K}(x)$, at least in the NF-case, is a special case of Weil's general explicit formula. To be precise, use $t$ for $x$ of $\left[\mathrm{W}_{1}\right]$, keeping $x$ for our $x$, and put

$$
F(t)= \begin{cases}\frac{1}{x-1}\left(x e^{-t / 2}-e^{t / 2}\right) & \cdots 0<t<\log x, \\ \frac{1}{2} & \cdots t=0, \\ 0 & \cdots \text { otherwise }\end{cases}
$$

in the formula $(11)$ of $\left[\mathrm{W}_{1}\right]$. Then we obtain (1.2.1) by straightforward computations. The FF-case is not fully treated in $\left[\mathrm{W}_{1}\right]$ (nor in $\left[\mathrm{W}_{2}\right]$ except when $t$ is an integral multiple of $\log q$ ), but this case is easier.

In this $\S 1.3$, we shall give a brief account of some basic materials for, and a sketch of,the proof of (1.2.1) valid in both cases, which, hopefully is enough for the readers to convince themselves of the validity also in the FF-case, and to see why the term $\phi(q, x)$ should appear. The formula (1.3.11) obtained in this process will anyway be needed later. The advanced readers can skip this section.

The explicit formula itself, and its connection with $\gamma_{K}$, both follow from the partial fraction decomposition of the logarithmic derivative of $\zeta_{K}(s)$. Put

$$
Z_{K}(s)=-\frac{\zeta_{K}^{\prime}(s)}{\zeta_{K}(s)}
$$

Then from the Euler product expansion

$$
\zeta_{K}(s)=\prod_{P}\left(1-N(P)^{-s}\right)^{-1} \quad(\operatorname{Re}(s)>1)
$$

of $\zeta_{K}(s)$ follows the Dirichlet series expansion

$$
Z_{K}(s)=\sum_{P, k \geq 1} \frac{\log N(P)}{N(P)^{k s}} \quad(\operatorname{Re}(s)>1)
$$

for $Z_{K}(s)$. In terms of $Z_{K}(s)$, the Euler-Kronecker constant $\gamma_{K}$ has the expression

$$
\gamma_{K}=-\lim _{s \rightarrow 1}\left(Z_{K}(s)-\frac{1}{s-1}\right) .
$$

This $Z_{K}(s)$ has the following partial fraction expansion ("Stark's lemma");

$$
Z_{K}(s)=\frac{1}{s}+\frac{1}{s-1}-\sum_{\rho} \frac{1}{s-\rho}+\alpha_{K}+\beta_{K}+\xi_{K}(s),
$$


with

$$
\begin{aligned}
\xi_{K}(s) & =\frac{r_{1}}{2}\left(g\left(\frac{s}{2}\right)-g\left(\frac{1}{2}\right)\right)+r_{2}(g(s)-g(1)) & \\
& =-r_{1}\left(\frac{1-s}{s}+\sum_{n=1}^{\infty}\left(\frac{1}{s+2 n}-\frac{1}{1+2 n}\right)\right)-r_{2}\left(\frac{1-s}{s}+\sum_{n=1}^{\infty}\left(\frac{1}{s+n}-\frac{1}{1+n}\right)\right) & (N F) \\
& =\sum_{\theta \neq 0,1} \frac{1}{s-\theta} & (F F),
\end{aligned}
$$

where $\rho$ runs over the non-trivial zeros of $\zeta_{K}(s), \theta$ runs over all poles $\neq 0,1$ of $\zeta_{K}(s)$ (FF-case),

$$
\sum_{\rho}=\lim _{T \rightarrow \infty} \sum_{|\rho|<T}, \quad \sum_{\theta}=\lim _{T \rightarrow \infty} \sum_{|\theta|<T}
$$

and

$$
g(s)=\frac{\Gamma^{\prime}(s)}{\Gamma(s)}
$$

(Note that $g(1)=-\gamma_{\mathbb{Q}}, g\left(\frac{1}{2}\right)=-\gamma_{\mathbb{Q}}-\log 4$.)

( In the NF-case, (1.3.5) is Stark's lemma [St](9) itself. The FF-case follows directly from the rational expression

$$
\zeta_{K}(s)=\prod_{\alpha \in A}\left(1-\alpha q^{-s}\right)^{\lambda_{\alpha}} \quad\left(A: \text { a finite subset of } \mathbb{C}^{\times}, \lambda_{\alpha}= \pm 1\right)
$$

of $\zeta_{K}(s)$

$$
\begin{aligned}
Z_{K}(s) & =\frac{-d \log \zeta_{K}(s)}{d s}=\sum_{\alpha \in A} \lambda_{\alpha} \frac{\log q}{1-\alpha^{-1} q^{s}} \\
& =\sum_{\alpha \in A} \lambda_{\alpha}\left(\frac{\log q}{2}-\sum_{q^{\beta}=\alpha} \frac{1}{s-\beta}\right) \quad(\operatorname{by}(1.2 .11)) \\
& \left.=(g-1) \log q-\sum_{\rho} \frac{1}{s-\rho}+\left(\frac{1}{s}+\frac{1}{s-1}+\sum_{\begin{array}{c}
\theta \neq 0,1 \\
\text { poles }
\end{array}} \frac{1}{s-\theta}\right) .\right)
\end{aligned}
$$

Now by combining (1.3.4) with (1.3.5), we obtain easily

$$
\begin{aligned}
\gamma_{K} & =\sum_{\rho} \frac{1}{\rho}-\alpha_{K}-\beta_{K}-c_{K} \\
& =\frac{1}{2} \sum_{\rho} \frac{1}{\rho(1-\rho)}-\alpha_{K}-\beta_{K}-c_{K},
\end{aligned}
$$

where $\alpha_{K}, \beta_{K}$ are as defined by (1.2.2),(1.2.3) respectively, and

$$
\begin{aligned}
c_{K} & =1 & & (N F), \\
& =c_{q}=\frac{q+1}{2(q-1)} \log q & & (F F) .
\end{aligned}
$$


The last formula for $c_{K}$ in the FF-case follows directly from (1.2.11) for $s=\alpha=1$, because $\xi_{K}(1)+1=\sum_{\substack{q^{\theta}=1, q \\ \theta \neq 1}}(1-\theta)^{-1}=\sum_{q^{\theta}=1}(1-\theta)^{-1}$.

Remark If we define $c_{q}$ for each $q \in \mathbb{R}, q>1$ by (1.3.12), then $c_{q}>1$ and $\lim _{q \rightarrow 1} c_{q}=1$. This matches with the well-known belief that "the constant field of a number field should be $\mathbb{F}_{1} "$.

The explicit formula (1.2.1) follows from the evaluation of the integral

$$
\Phi_{K}^{(\mu)}(x)=\frac{1}{2 \pi i} \int_{c-i \infty}^{c+i \infty} \frac{x^{s-\mu}}{s-\mu} Z_{K}(s) d s \quad(c \gg 0)
$$

for $\mu=0$ and 1 in two ways, based on the classical formula

$$
\frac{1}{2 \pi i} \int_{c-i \infty}^{c+i \infty} \frac{y^{s}}{s} d s=\left\{\begin{array}{lll}
0 & \cdots & 0<y<1 \\
1 / 2 & \cdots & y=1 \\
1 & \cdots & 1<y
\end{array}\right.
$$

The Dirichlet series expansion (1.3.3) of $Z_{K}(s)$ gives the connection

$$
x \Phi_{K}^{(1)}(x)-\Phi_{K}^{(0)}(x)=(x-1) \Phi_{K}(x),
$$

while the partial fraction decomposition (1.3.5) of $Z_{K}(s)$ gives, via standard residue calculations,

$$
x \Phi_{K}^{(1)}(x)-\Phi_{K}^{(0)}(x)=(x-1)\left\{\log x+\left(\alpha_{K}+\beta_{K}\right)+\ell_{K}(x)+r_{K}(x)\right\} .
$$

The terms $\log x, \ell_{K}(x)$ and $r_{K}(x)$ inside \{\} , correspond to

$$
\frac{1}{s}+\frac{1}{s-1}, \quad \xi_{K}(s), \quad \text { and } \quad-\sum_{\rho} \frac{1}{s-\rho}
$$

in (1.3.5), respectively.

Remarks (i) A word about the constant $\beta_{K}$ (NF-case). If

$$
\Gamma_{\mathbb{R}}(s)=\pi^{-\frac{s}{2}} \Gamma\left(\frac{s}{2}\right), \text { resp. } \Gamma_{\mathbb{C}}(s)=(2 \pi)^{-s} \Gamma(s)
$$


denote the standard $\Gamma$-factors at the real (resp. imaginary) places, so that

$$
\Lambda_{K}(s)=\Gamma_{\mathbb{R}}(s)^{r_{1}} \Gamma_{\mathbb{C}}(s)^{r_{2}} \zeta_{K}(s)
$$

satisfies the functional equation

$$
\Lambda_{K}(s)=\left(\sqrt{\left|d_{K}\right|}\right)^{1-2 s} \Lambda_{K}(1-s)
$$

then one has

$$
\begin{gathered}
\left(\frac{d \log \Gamma_{\mathbb{R}}(s)}{d s}\right)_{s=1}=-\frac{1}{2}\left(\gamma_{\mathbb{Q}}+\log 4 \pi\right), \\
\left(\frac{d \log \Gamma_{\mathbb{C}}(s)}{d s}\right)_{s=1}=-\left(\gamma_{\mathbb{Q}}+\log 2 \pi\right) .
\end{gathered}
$$

Therefore,

$$
\lim _{s \rightarrow 1}\left(\left(\frac{d \log \Lambda_{K}(s)}{d s}\right)+\frac{1}{s-1}\right)=\gamma_{K}+\beta_{K} .
$$

Thus, $\beta_{K}$ is the "archimedean counterpart" of $\gamma_{K}$.

(ii) Incidentally, the functional equation in the function field case for $\Lambda_{K}(s)=\zeta_{K}(s)$ is

$$
\Lambda_{K}(s)=\left(q^{g-1}\right)^{1-2 s} \Lambda_{K}(1-s),
$$

and the comparison of (1.3.18) and (1.3.22) leads to our common recognition that the FF-analogue of $\frac{1}{2} \log |d|$ should be $(g-1) \log q$. In both cases, the constant term in the partial fraction decomposition of $Z_{K}(s)$ is determined from the functional equation.

\subsection{Some elementary formulas related to $\gamma_{K}$}

We shall give a few more remarks related to the quantity

$$
\gamma_{K}=\sum_{\rho} \frac{1}{\rho}-\alpha_{K}-\beta_{K}-c_{K}
$$

When $\alpha_{K}$ is large, each of $\sum_{\rho} \rho^{-1}$ and $\alpha_{K}+\beta_{K}$ is usually much larger than the absolute value of $\gamma_{K}$. (Only for some special families of $K$, they have the same order of magnitude; see $\S 1.6$.) So, $\gamma_{K}$ is a finer object for study than $\sum_{\rho} \rho^{-1}$.

In the FF-case, in terms of the reciprocal roots $\pi_{\nu}, \bar{\pi}_{\nu}(1 \leq \nu \leq g)$ of $\zeta_{K}(s)$ in $u=q^{-s}$, we have (as is obvious by (1.2.12))

$$
\sum_{\rho} \frac{1}{\rho}=(q-1) \sum_{\nu=1}^{g} \frac{1}{\left(\pi_{\nu}-1\right)\left(\bar{\pi}_{\nu}-1\right)} \log q ;
$$


hence

$$
\begin{aligned}
\gamma_{K} & =\left\{(q-1) \sum_{\nu=1}^{g} \frac{1}{\left(\pi_{\nu}-1\right)\left(\bar{\pi}_{\nu}-1\right)}-(g-1)\right\} \log q-c_{q} \\
& =\sum_{\nu=1}^{g}\left(\frac{1}{\pi_{\nu}-1}+\frac{1}{\bar{\pi}_{\nu}-1}\right) \log q+\left(\log q-c_{q}\right) \\
& =\sum_{m=1}^{\infty} \sum_{\nu=1}^{g}\left(\pi_{\nu}^{m}+\bar{\pi}_{\nu}^{m}\right) q^{-m} \log q+\left(\log q-c_{q}\right) \\
& =\sum_{m=1}^{\infty}\left(q^{m}+1-N_{m}\right) q^{-m} \log q+\left(\log q-c_{q}\right) .
\end{aligned}
$$

Consider the arithmetic, geometric, and harmonic means of $g$ positive real numbers

$$
\left(\pi_{\nu}-1\right)\left(\bar{\pi}_{\nu}-1\right) \quad(1 \leq \nu \leq g) .
$$

Then if $X$ denotes the proper smooth curve over $\mathbb{F}_{q}$ corresponding to $K$, and $J$ its Jacobian, the above three means of (1.4.4) are given respectively by

$$
\begin{aligned}
& \text { a.m. }=\frac{1}{g} \# X\left(\mathbb{F}_{q}\right)+\left(1-\frac{1}{g}\right)(q+1) \\
& \vee \mathrm{I} \\
& \text { g.m. }=\left(\# J\left(\mathbb{F}_{q}\right)\right)^{1 / g} \\
& \mathrm{VI} \\
& \text { h.m. }=\frac{g(q-1) \log q}{\gamma_{K}+\alpha_{K}+c_{q}} .
\end{aligned}
$$

The three properties $\# X\left(\mathbb{F}_{q}\right)$ large, $\# J\left(\mathbb{F}_{q}\right)$ large, and $-\gamma_{K}$ large, are different but correlated, and are in a sense in the same direction. (The denominator of $h . m$. is $\sum_{\rho} \rho^{-1}>$ 0.)

By the Riemann hypothesis for curves, we have

$$
(\sqrt{q}-1)^{2} \leq\left(\pi_{\nu}-1\right)\left(\bar{\pi}_{\nu}-1\right) \leq(\sqrt{q}+1)^{2} ;
$$

hence, by (1.4.3), we obtain immediately

$$
\left(\frac{-2 g}{\sqrt{q}+1}+1\right) \log q \leq \gamma_{K}+c_{q} \leq\left(\frac{2 g}{\sqrt{q}-1}+1\right) \log q .
$$

Later, we shall obtain much better bounds (§1.6). In particular, when $g$ is fixed and $q \rightarrow \infty$ (e.g. the constant field extensions), we have the limit formula

$$
\lim _{q \rightarrow \infty} \frac{\gamma_{K}}{\log q}=\frac{1}{2}
$$


When $g=0, \gamma_{K}$ is given by the equality

$$
\gamma_{K}+c_{q}=\log q
$$

(See Remark (i) below).

Remarks (i) We defined $\gamma_{K}$ as a natural generalization of the Euler-Mascheroni constant $\gamma_{\mathbb{Q}}$. But, in a sense, the quantity $\gamma_{K}+c_{K}$ may be more canonical, as some of the preceding formulas indicate! The quantities obtained by (further) adding $\beta_{K}(\mathrm{NF})$ or $-\log q(\mathrm{FF})$ can sometimes be better.

(ii) In the FF-case, if we use other poles of $\zeta_{K}(s)$, instead of $s=1$, to define $\gamma_{K}$ similarly, then what we obtain is still $\gamma_{K}$ if the pole is congruent to 1 modulo $2 \pi i / \log q$, and is $-\alpha_{K}-\gamma_{K}$ if the pole is $0 \bmod (2 \pi i / \log q)$.

In the NF-case, the order of zero at $s=0$ of $\zeta_{K}(s)$ is $r_{1}+r_{2}-1$, and

$$
\gamma_{K}=-\gamma_{K}^{\prime}-\log \left|d_{K}\right|+[K: \mathbb{Q}]\left(\gamma_{\mathbb{Q}}+\log (2 \pi)\right),
$$

where $\gamma_{K}^{\prime}$ is the coefficient of $s^{r_{1}+r_{2}}$ divided by that of $s^{r_{1}+r_{2}-1}$ in the Taylor expansion of $\zeta_{K}(s)$ at $s=0$.

\subsection{Estimations of $r_{K}(x)$ and $\ell_{K}(x)$}

Now we return to the explicit formula (1.2.1). By (1.3.11), one may rewrite it as

$$
\begin{aligned}
\log x-\Phi_{K}(x) & =-\left(\alpha_{K}+\beta_{K}\right)-r_{K}(x)-\ell_{K}(x) \\
& =\gamma_{K}+c_{K}-\left(r_{K}(x)+\sum_{\rho} \rho^{-1}\right)-\ell_{K}(x) \\
& =\gamma_{K}+c_{K}-\left(r_{K}(x)+\frac{1}{2} \sum_{\rho} \frac{1}{\rho(1-\rho)}\right)-\ell_{K}(x) .
\end{aligned}
$$

We are going to estimate the non-constant terms on the right side of (1.5.1). In most of what follows, we shall assume GRH (which is satisfied in the FF-case).

Main Lemma $((\mathrm{FF})$; and $(\mathrm{NF})$ under $(\mathrm{GRH}))$ For any $x>1$ we have

$$
\frac{\sqrt{x}-1}{\sqrt{x}+1}\left(\frac{1}{2} \sum_{\rho} \frac{1}{\rho(1-\rho)}\right) \leq-r_{K}(x) \leq \frac{\sqrt{x}+1}{\sqrt{x}-1}\left(\frac{1}{2} \sum_{\rho} \frac{1}{\rho(1-\rho)}\right) .
$$

Proof Since

$$
-r_{K}(x)=\frac{1}{2} \sum_{\rho}\left\{\frac{\left(x^{\rho}-1\right)\left(x^{1-\rho}-1\right)}{(x-1)} \cdot \frac{1}{\rho(1-\rho)}\right\}
$$

and $\rho=\frac{1}{2}+i \gamma(\gamma \in \mathbb{R})$, it follows that $\rho(1-\rho)=\frac{1}{4}+\gamma^{2}>0$, and that

$$
\left(x^{\rho}-1\right)\left(x^{1-\rho}-1\right)=x+1-2 \sqrt{x} \cos (\gamma \log x)
$$

lies in-between $(\sqrt{x}-1)^{2}$ and $(\sqrt{x}+1)^{2}$; whence our inequalities. 
The graphs of the three functions of $x$ appearing in (1.5.2) in the Main lemma, for the cases $K=\mathbb{Q}, \mathbb{Q}(\sqrt{481})$, are as shown in Figures $1.5 A_{1}, A_{2}$.

(As (1.5.3) indicates, each $\rho$ with small $|\gamma|$ contributes to a "high wave calm on the surface", whereas a larger $|\gamma|$, to a lower "ripple". The effect of the first few $\rho$ is not particularly large, but sometimes determines the main shape of the graph (for $x$ not too large). Thus, these graphs seem to indicate that the smallest $|\gamma|$ for $\mathbb{Q}(\sqrt{481})$ would be much smaller than that of $K=\mathbb{Q}$ (i.e., 14.1347...).)

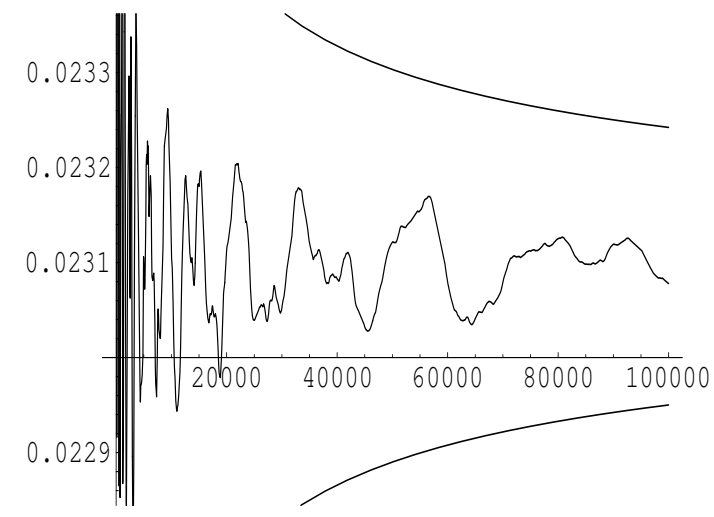

Figure $1.5 A_{1}: K=\mathbb{Q}$

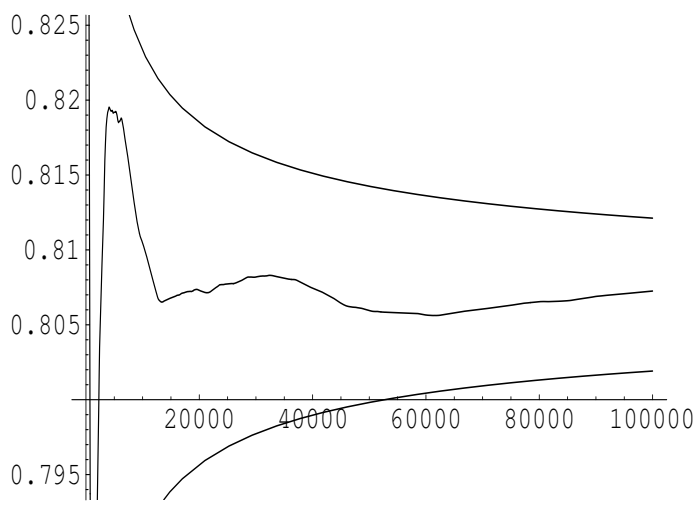

Figure $1.5 A_{2}: K=\mathbb{Q}(\sqrt{481})$

By this lemma and (1.3.11) we obtain

$\frac{-2}{\sqrt{x}+1}\left(\gamma_{K}+\alpha_{K}+\beta_{K}+c_{K}\right) \leq-r_{K}(x)-\frac{1}{2} \sum_{\rho} \frac{1}{\rho(1-\rho)} \leq \frac{2}{\sqrt{x}-1}\left(\gamma_{K}+\alpha_{K}+\beta_{K}+c_{K}\right)$,

and hence by (1.5.1)

$$
\begin{aligned}
\frac{\sqrt{x}-1}{\sqrt{x}+1}\left(\gamma_{K}+c_{K}\right)-\frac{2}{\sqrt{x}+1}\left(\alpha_{K}+\beta_{K}\right)-\ell_{K}(x) & \leq \log x-\Phi_{K}(x) \\
& \leq \frac{\sqrt{x}+1}{\sqrt{x}-1}\left(\gamma_{K}+c_{K}\right)+\frac{2}{\sqrt{x}-1}\left(\alpha_{K}+\beta_{K}\right)-\ell_{K}(x)
\end{aligned}
$$

(under GRH).

As for $\ell_{K}(x)$, we have

Lemma 2 (i). (NF-case) $\quad \ell_{K}(x)$ is monotone decreasing,

$$
\lim _{x \rightarrow 1} \ell_{K}(x)=+\infty, \quad \lim _{x \rightarrow \infty} \ell_{K}(x)=0,
$$

and

$$
0<\ell_{K}(x)<[K: \mathbb{Q}]\left(\frac{\log x+1}{x-1}\right) \quad(x>1) .
$$


(ii). (FF-case) $\quad \ell_{K}(x)=0$ if and only if $x=q^{m}(m \in \mathbb{N})$; for other $x, \ell_{K}(x)$ always remains within the open interval $(0, \log q)$, but does not tend to 0 as $x \rightarrow \infty$.

Proof (i)(NF-case). In this case,

$$
\left\{\begin{array}{l}
\ell_{K}(x)=\frac{r_{1}}{2} F_{1}(x)+r_{2} F_{2}(x), \text { with } \\
F_{1}(x)=\log \frac{x+1}{x-1}+\frac{2}{x-1} \log \frac{x+1}{2} \\
F_{2}(x)=\log \frac{x}{x-1}+\frac{1}{x-1} \log x
\end{array}\right.
$$

First, since $F_{1}^{\prime}(x)=-2(x-1)^{-2} \log ((x+1) / 2)<0, F_{1}(x)$ is monotone decreasing. Secondly, since $F_{2}(x)=F_{1}(2 x-1), F_{2}(x)$ is also monotone decreasing and $F_{2}(x)<F_{1}(x)$. Thirdly, since $\log \left(\frac{x+1}{x-1}\right)<2(x-1)^{-1}$ and $\log \left(\frac{x+1}{2}\right)<\log x$, we obtain

$$
F_{1}(x)<2(\log x+1)(x-1)^{-1},
$$

and it is clear that $F_{2}(x)>0$. The desired inequalities follow immediately from these. The assertions for the limits at $x \rightarrow 1, \infty$ of $\ell_{K}(x)$ are also obvious. (The following inequality will be used later $(\S 2.4)$;

$$
\left.\frac{1}{2}(x-1) F_{1}(x)=\log (x+1)+\log \left[\frac{1}{2}\left(1+\frac{2}{x-1}\right)^{\frac{x-1}{2}}\right] \geq \log (x+1) \quad(x \geq 3) .\right)
$$

(ii)(FF-case). We already know that $\phi(q, x)=0$ if $x=q^{m}(m \in \mathbb{N})$. So, put $x=q^{m-1+y}$, with $m \geq 1,0<y<1$. Then by $(1.2 .15)$,

$$
\begin{aligned}
\phi(q, x) & =\left(1-y-\frac{\left(q^{m-1}-1\right)\left(q-q^{y}\right)}{\left(q^{m-1+y}-1\right)(q-1)}\right) \log q \\
& =\left(\frac{\left(q^{m}-1\right)\left(q^{y}-1\right)}{\left(q^{m-1+y}-1\right)(q-1)}-y\right) \log q .
\end{aligned}
$$

It is easy to see that if we fix $y$, then this is monotone decreasing as a function of $m$, and tends uniformly to

$$
s_{q}(y)=\left(\frac{1-q^{-y}}{1-q^{-1}}-y\right) \log q
$$

as $m \rightarrow \infty$. Therefore,

$$
0<\frac{1-q^{-y}}{1-q^{-1}}-y<\frac{\phi(q, x)}{\log q} \leq 1-y<1
$$

which proves all the assertions stated in Lemma 2 (ii). 
Remark Note that $s_{q}(0)=s_{q}(1)=0, s_{q}(y)>0$ for $0<y<1$. The maximal value of $s_{q}(y)$ for $0<y<1$ is

$$
\frac{\log q}{1-q^{-1}}-\left(\log \log q-\log \left(1-q^{-1}\right)+1\right)
$$

which is attained at

$$
y=\frac{\log \log q-\log \left(1-q^{-1}\right)}{\log q} .
$$

The graphs of $F_{1}(x)>F_{2}(x)$ will be shown in Figure $1.5 B_{1}$, and that of $\phi\left(q, q^{z}\right)$ for $q=5$, in Figure $1.5 B_{2}$. The horizontal line in the latter gives the value of (1.5.13) for $q=5$.

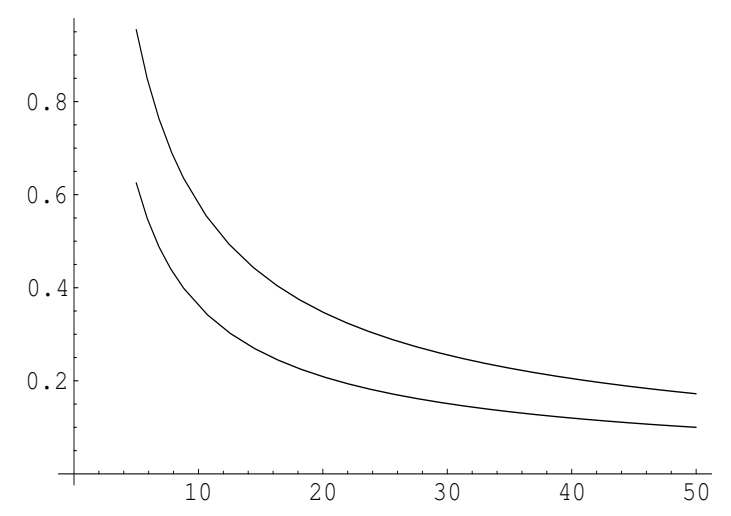

Figure $1.5 B_{1}$ :

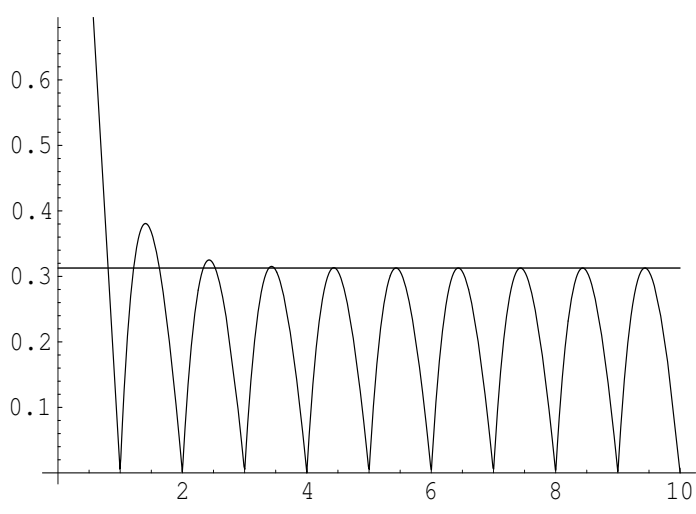

Figure $1.5 B_{2}$ :

\subsection{Estimations related to $\gamma_{K}$}

From (1.5.6) we obtain immediately:

Proposition 2 (Under $(\mathrm{GRH})$ in the $(\mathrm{NF})$ case) For any $x>1$ we have

$$
\begin{aligned}
& \gamma_{K} \leq \frac{\sqrt{x}+1}{\sqrt{x}-1}\left(\log x-\Phi_{K}(x)+\ell_{K}(x)\right)+\frac{2}{\sqrt{x}-1}\left(\alpha_{K}+\beta_{K}\right)-c_{K}, \\
& \gamma_{K} \geq \frac{\sqrt{x}-1}{\sqrt{x}+1}\left(\log x-\Phi_{K}(x)+\ell_{K}(x)\right)-\frac{2}{\sqrt{x}+1}\left(\alpha_{K}+\beta_{K}\right)-c_{K} .
\end{aligned}
$$

Since by (1.5.6) the difference between the upper and the lower bounds tends to 0 as $x \rightarrow \infty$, this gives a method for computing the constant $\gamma_{K}$ (under GRH) to as much accuracy as one desires. Although the convergence is slow, one can usually determine the approximate size of $\gamma_{K}$ (e.g. its sign) even by hand calculations. 


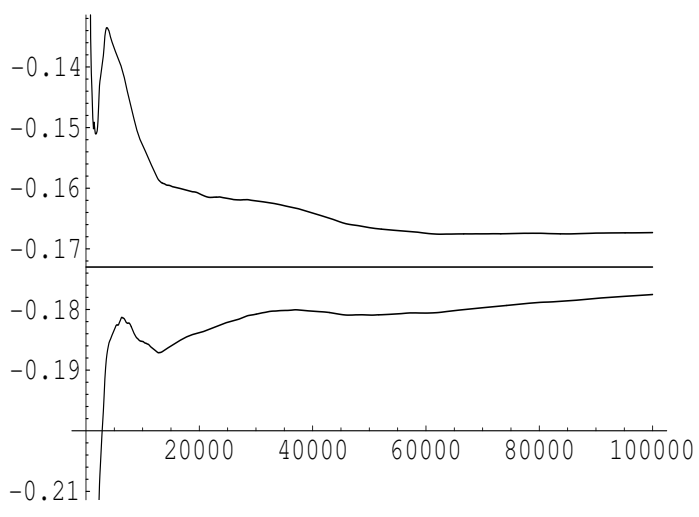

Figure 1.6 $A_{1}: K=\mathbb{Q}(\sqrt{481})$

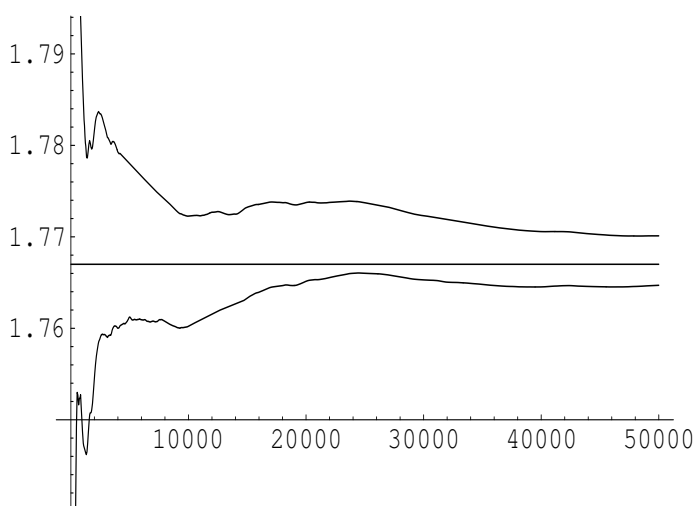

Figure $1.6 A_{2}: K=\mathbb{Q}\left(\cos \frac{2 \pi}{9}\right)$

Figures $1.6 A_{1}, 1.6 A_{2}$ show two examples for the graphs of the upper and the lower

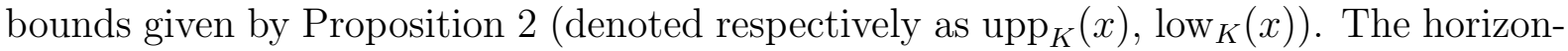
tal lines indicate the expected values of $\gamma_{K}$.

Examples(by computer). Let $K=\mathbb{Q}(\sqrt{-1})$, and take $x=50,000$. Then the upper and the lower bounds for $\gamma_{K}$ given by Proposition 2 (i)(ii) are 0.8239498, 0.8221413, respectively. The value of $\gamma_{K}$ computed by using the Kronecker formula (cf. §2.2) is 0.82282525 . Incidentally, in this case, the value of $\log x-\Phi_{K}(x)-1$ is 0.82280515 which is close to the actual value, and lies in between the above upper and lower bounds. But in general, $\log x-\Phi_{K}(x)-1$ need not lie in between the two bounds of Proposition 2 (see Remark (ii) below).

For other imaginary quadratic fields, $0<\gamma_{K}<1$ holds for $\left|d_{K}\right| \leq 43$, but $\gamma_{K}<0$ for $d_{K}=-47,-56, \cdots$. For example,

$$
-0.072<\gamma_{\mathbb{Q}(\sqrt{-47})}<-0.053 .
$$

For real quadratic fields, $0<\gamma_{K}<2$ for $d_{K}<100$, but

$$
-0.181<\gamma_{\mathbb{Q}(\sqrt{481})}<-0.167 .
$$

These are, of course, under (GRH).

Some other examples will be given in $\S 1.7$ and $\S 2.3$.

We shall give some applications. First, by letting $x \rightarrow \infty$ in (1.5.6) we obtain

\section{Corollary 1}

$$
\gamma_{K}=\lim _{x \rightarrow \infty}\left(\log x-\Phi_{K}(x)-1\right)
$$




$$
\gamma_{K}=\lim _{\substack{m \rightarrow \infty \\ m \in \mathbb{N}}}\left(\log \left(q^{m}\right)-\Phi_{K}\left(q^{m}\right)-c_{q}\right)
$$

A formula equivalent to (1.6.1) can be found also in a recent preprint [HIKW](Th. B).

Remarks (i) For (1.6.1), GRH is unnecessary. In fact, by using a standard zero-free region for $\zeta_{K}(s)$, one can show, unconditionally, that

$$
\lim _{x \rightarrow \infty}\left\{r_{K}(x)+\frac{1}{2} \sum_{\rho} \frac{1}{\rho(1-\rho)}\right\}=0,
$$

from which (1.6.1) follows directly by (1.5.1) and Lemma 2. The proof of (1.6.3) runs as follows. As is well-known (cf. e.g. [L-O] lemma 8.1), there exists a positive constant $c$ (depending on $K$ ) such that if $\rho=\beta+i \gamma$ is a non-trivial zero of $\zeta_{K}(s)$ with $|\gamma|$ sufficiently large, then

$$
\beta<1-c(\log |\gamma|)^{-1} .
$$

We claim that

$$
\lim _{x \rightarrow \infty}\left(\sum_{\rho} \frac{x^{\beta-1}}{\gamma^{2}}\right)=0,
$$

where $\rho$ runs over all imaginary zeros of $\zeta_{K}(s)$. To show this, since $\beta<1$, we may exclude finitely many $\rho$ 's and assume that (1.6.4) is satisfied. Then, for $x>1$,

$$
\sum_{\rho} \frac{x^{\beta-1}}{\gamma^{2}}<\sum_{\rho} \frac{x^{-c(\log |\gamma|)^{-1}}}{\gamma^{2}}=\sum_{\log |\gamma|<T}+\sum_{\log |\gamma| \geq T}
$$

where we choose $T=\sqrt{\log x}$. Then

$$
\sum_{\log |\gamma|<T} \leq\left(\sum_{\log |\gamma|<T} \frac{1}{\gamma^{2}}\right) x^{-c T^{-1}} \leq\left(\sum_{\rho} \frac{1}{\gamma^{2}}\right) \exp (-c \sqrt{\log x}) \rightarrow 0,
$$

and

$$
\sum_{\log |\gamma| \geq T} \leq \sum_{\log |\gamma| \geq \sqrt{\log x}} \frac{1}{\gamma^{2}} \rightarrow 0
$$

whence (1.6.5). But since

$$
r_{K}(x)+\frac{1}{2} \sum_{\rho} \frac{1}{\rho(1-\rho)}=\frac{1}{2} \sum_{\rho} \frac{1}{\rho(1-\rho)}\left(\frac{x^{\rho}+x^{1-\rho}-2}{x-1}\right),
$$

and each term tends to 0 as $x \rightarrow \infty$, by (1.6.5), we obtain

$$
\lim _{x \rightarrow \infty}\left|r_{K}(x)+\frac{1}{2} \sum_{\rho} \frac{1}{\rho(1-\rho)}\right| \leq \lim _{x \rightarrow \infty} \sum_{\substack{\rho, \gamma \neq 0}} \frac{1}{\gamma^{2}} \frac{x^{\beta}}{x-1}=0 .
$$


(ii) Some readers may be interested in the comparison between $\mathrm{A}_{K}(x):=\log x-\Phi_{K}(x)-1$ and the two bounds $\operatorname{upp}_{K}(x), \operatorname{low}_{K}(x)$ of Proposition 2. From (1.2.1) we obtain easily

$$
\begin{aligned}
& \frac{\sqrt{x}-1}{2}\left(\operatorname{upp}_{K}(x)-\mathrm{A}_{K}(x)\right)=-r_{K}(x)+\frac{\sqrt{x}-1}{2} \ell_{K}(x), \\
& \frac{\sqrt{x}+1}{2}\left(\mathrm{~A}_{K}(x)-\operatorname{low}_{K}(x)\right)=-r_{K}(x)-\frac{\sqrt{x}+1}{2} \ell_{K}(x),
\end{aligned}
$$

and we moreover have $-r_{K}(x)>0$ under GRH. Therefore, $\mathrm{A}_{K}(x)<\operatorname{upp}_{K}(x)$ always holds under GRH. But $\mathrm{A}_{K}(x)>\operatorname{low}_{K}(x)$ need not hold in general ; a counterexample being given by $K=\mathbb{Q}(\sqrt{-1}), x=$ (say) 1800 .

[Examples of graphs of $\left.\mathrm{A}_{K}(x)=\log x-\Phi_{K}(x)-1\right]$

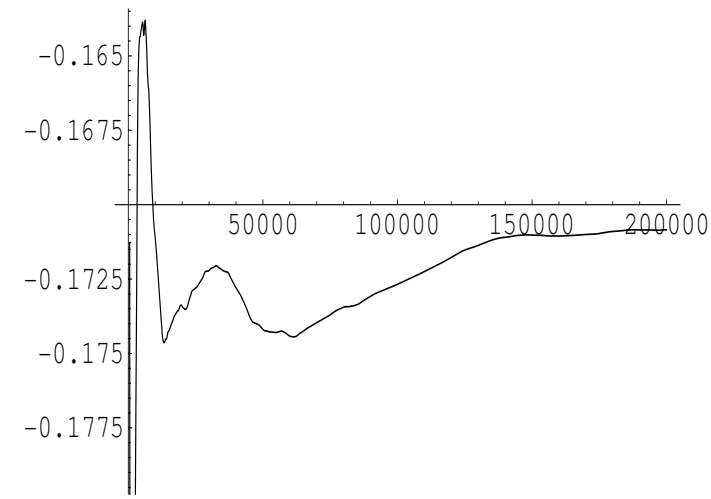

Figure $1.6 B_{1}: K=\mathbb{Q}(\sqrt{481})$

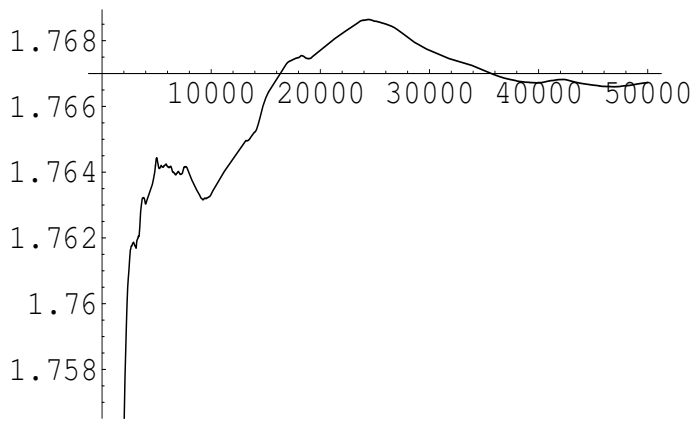

Figure $1.6 B_{2}: K=\mathbb{Q}\left(\cos \frac{2 \pi}{9}\right)$

[Upper bounds] The second application of Proposition 2 is to the problem of finding a reasonably good general upper bound for $\gamma_{K}$ in terms of more elementary invariants of $K$. It can be obtained from Proposition 2(i) by the substitution of a suitable value of $x$. Since we do not know a priori the local behavior of $\Phi_{K}(x)$, except that $\Phi_{K}(x) \geq 0$, what we do is try to minimize

$$
\frac{\sqrt{x}+1}{\sqrt{x}-1}\left(\log x+\ell_{K}(x)\right)+\frac{2}{\sqrt{x}-1}\left(\alpha_{K}+\beta_{K}\right)-c_{K}
$$

We leave the discussion of this delicate question until a little later (after the proof of Theorem 1), and first see what we can obtain by choosing the value $x_{0}$ of $x$ which minimizes

$$
\log x+\frac{2 \alpha_{K}}{\sqrt{x}}
$$

i.e., $x_{0}=\alpha_{K}^{2}$. We then obtain 
Theorem 1 (Under (GRH) in the (NF)-case)

$$
\begin{aligned}
\gamma_{K} & <\left(\frac{\alpha_{K}+1}{\alpha_{K}-1}\right)\left(2 \log \alpha_{K}+a-\Phi_{K}\left(\alpha_{K}^{2}\right)\right) \\
& \leq\left(\frac{\alpha_{K}+1}{\alpha_{K}-1}\right)\left(2 \log \alpha_{K}+a\right)
\end{aligned}
$$

provided that

$$
\begin{array}{lll}
g>2, \text { or } & g=2 \text { and } q>2 & (F F) \\
n>2, \text { or } & n=2 \text { and }\left|d_{K}\right|>8 & (N F) .
\end{array}
$$

Here, $a=1$ and $n=[K: \mathbb{Q}](\mathrm{NF}), \quad a=1+\log q(\mathrm{FF})$.

Proof The right hand side of Proposition 2(i) for $x=\alpha_{K}^{2}$ can be rewritten as

$$
\begin{cases}\frac{\alpha_{K}+1}{\alpha_{K}-1}\left(2 \log \alpha_{K}-\Phi_{K}\left(\alpha_{K}^{2}\right)+1\right)+\frac{1}{\alpha_{K}-1}\left(\left(\alpha_{K}+1\right) \ell_{K}\left(\alpha_{K}^{2}\right)+2 \beta_{K}\right) & (N F), \\ \frac{\alpha_{K}+1}{\alpha_{K}-1}\left(2 \log \alpha_{K}-\Phi_{K}\left(\alpha_{K}^{2}\right)+1+\phi\left(q, \alpha_{K}^{2}\right)\right)+\left(1-c_{q}\right) & (F F) .\end{cases}
$$

In the FF-case, (1.6.9) implies $\alpha_{K}>1$. And since $\phi\left(q, \alpha_{K}^{2}\right)<\log q$ and $1-c_{q}<0$, we are done. In the NF-case, we have the following inequalities;

$$
\left\{\begin{array}{l}
f_{1}(x):=(x+1)\left\{\log \left(\frac{x^{2}+1}{x^{2}-1}\right)+\frac{2}{x^{2}-1} \log \left(\frac{x^{2}+1}{2}\right)\right\}-2\left(\gamma_{\mathbb{Q}}+\log 4 \pi\right)<0 \quad \text { for } \quad x>1.16, \\
f_{2}(x):=(x+1)\left\{\log \left(\frac{x^{2}}{x^{2}-1}\right)+\frac{1}{x^{2}-1} \log \left(x^{2}\right)\right\}-2\left(\gamma_{\mathbb{Q}}+\log 2 \pi\right)<0 \quad \text { for } \quad x>1.16 .
\end{array}\right.
$$

They hold because $f_{1}(x), f_{2}(x)$ are both monotone decreasing for $x>1$, and their values at 1.16 are both negative (being $-0.08762 \ldots,-0.03882 \ldots$,respectively). Therefore,

$$
\left(\alpha_{K}+1\right) \ell_{K}\left(\alpha_{K}^{2}\right)+2 \beta_{K}<0 \text { for } \alpha_{K}>1.16 \text {. }
$$

But even the Minkowski lower bound for $\left|d_{K}\right|$ shows that $\alpha_{K}>1.16$ holds for $n>2$ and for $n=2$ with $\left|d_{K}\right|>10.2$ (which is actually the same as $\left|d_{K}\right|>8$ ).

[Discussions on minimizing (1.6.7)] Write $x=t^{2}(t>1)$, and put

$$
s=s_{K}=\alpha_{K}+\beta_{K} .
$$

Then (1.6.7) can be expressed as

$$
g(t)=g_{1}(t)+g_{2}(t)
$$

with

$$
g_{1}(t):=\frac{2}{t-1}((t+1) \log t+s)-c_{K}
$$




$$
\begin{aligned}
g_{2}(t): & =\frac{t+1}{t-1}\left\{\frac{r_{1}}{2} F_{1}\left(t^{2}\right)+r_{2} F_{2}\left(t^{2}\right)\right\} & & (>0)(N F) \\
& =\frac{t+1}{t-1} \phi\left(q, t^{2}\right) & & (\geq 0)(F F)
\end{aligned}
$$

(cf.(1.5.7)). These are continuous functions of $t>1$ parametrized by $s(\in \mathbb{R}) ; r_{1}, r_{2} \geq 0$, $r_{1}+r_{2}>0(\mathrm{NF})$, or $q(\mathrm{FF})$. We shall exclude the trivial case of genus $0(\mathrm{FF})$. Then $g(t)$ always achieves its minimal value at some $\theta(1<\theta<\infty)$, because it is continuous and tends to $+\infty$ at both ends, i.e., $t \rightarrow 1$ and $t \rightarrow \infty$.

[NF-case] In this case, $\theta$ is unique, as $(t-1)^{2} g^{\prime}(t)$ is monotone increasing. Indeed,

$$
(t-1)^{2} g_{1}^{\prime}(t)=2\left(t-2 \log t-t^{-1}-s\right)
$$

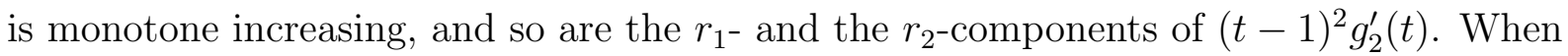
$s>1, \theta$ is close to

$$
s+2\left(1+\frac{r_{1}+r_{2}}{s}\right) \log s .
$$

We have included the $\left(r_{1}+r_{2}\right) s^{-1}$ term, because when $s_{K} \rightarrow \infty$, the quantity $\left(r_{1}+r_{2}\right) s_{K}^{-1}$, though bounded (by a standard unconditional lower bound for $\left|d_{K}\right|$ ), does not tend to 0 .

[FF-case] The local differential structure of $g(t)$ is, in a sense, opposite to the NFcase. The graph of $g(t)$ looks like a bouncing ball, bouncing at each integral power of $\sqrt{q}$, first coming down a slope and then going up another forever. (The slopes correspond to the graph of $g_{1}(t)$.) Indeed, $(t-1)^{2} g^{\prime}(t)$ is a negative constant $-2 g \log q$ ( $g$ : the genus) on $1<t^{2}<q$, and is monotone decreasing on every open interval $q^{m-1}<t^{2}<q^{m}(m>1)$. (The derivative of $(t-1)^{2} g^{\prime}(t)$ on this interval is $-2 a(t-1)^{-2}$, where

$$
\left.a=\left(q^{m-1}-1\right)\left(q^{m}-1\right)\left(q^{m}-q^{m-1}\right)^{-1} \log q .\right)
$$

Therefore, $g^{\prime \prime}(t)<0$ wherever $g^{\prime}(t)=0$. Therefore, $g(t)$ can acquire its minimal value only at the bouncing points $t \in(\sqrt{q})^{\mathbb{Z}}$. Note that $\phi\left(q, t^{2}\right)=0$ at bouncing points. Moreover, by (1.6.17), we conclude that $\theta$ must be one of the (at most two) integral powers of $\sqrt{q}$ which are adjacent to the unique root of the equation

$$
t-2 \log t-t^{-1}=s .
$$

Thus, again, $\theta$ is close to

$$
s+2 \log s
$$

as long as $s$ is large compared with $q$. If, on the other extreme,

$$
g<\frac{1}{\log q}\left(\sqrt{q}-\frac{1}{\sqrt{q}}\right)
$$


so that the root of (1.6.19) is smaller than $\sqrt{q}$, then $\theta$ is always equal to $\sqrt{q}$, and this gives rise only to the trivial general upper bound (1.4.6) for $\gamma_{K}$.

Each of Figures $1.6 C_{1}, 1.6 C_{2}$ gives the graphs of two functions

$$
g(t) \geq g_{1}(t)
$$

when $K=\mathbb{Q}(\sqrt{-5003}$ ) (Figure 1.6C $)$, and $q=2, g=5$ for $t^{2}=q^{y}$ (the horizontal axis is for $y$ ) (Figure $1.6 C_{2}$ ).

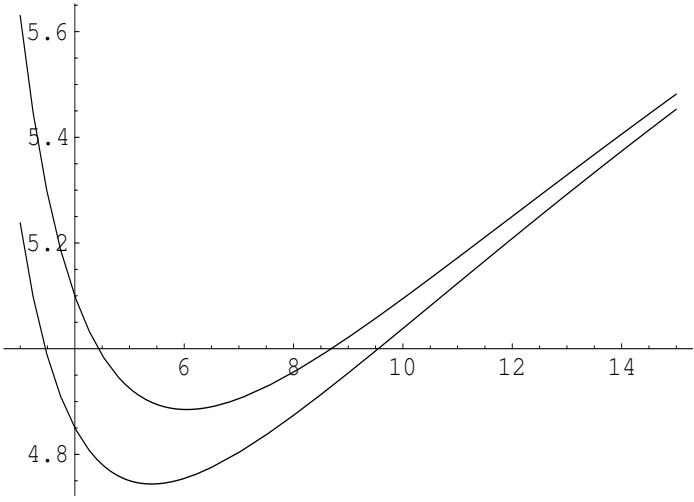

Figure $1.6 C_{1}$ :

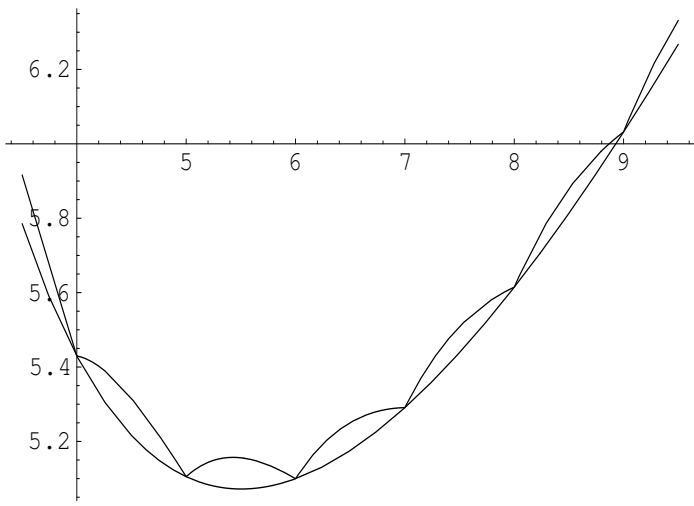

Figure $1.6 C_{2}$ :

Thus, the best possible approximation of $\theta$ would depend on the specific family of $K$ and purpose of applications. For example, the $\beta_{K}$-part of $s_{K}$ in the NF-case should not be neglected if $\left(r_{1}+r_{2}\right) s_{K}^{-1}$ is not small. But here, we are satisfied with having given a basic result expressed simply in terms of $\alpha_{K}$ only, together with some indications for possible improvements. We note that choices of other $\theta$ can improve only minor terms in Theorem 1, unless we restrict ourselves to some special families of $K$. Further studies of the upperbounds of $\gamma_{K}$ for various families of $K$ will be left to future publications.

The author has yet no idea about the minimal possible size of $\Phi_{K}\left(\theta^{2}\right)$ for each given $r_{1}, r_{2}$ and given approximate range of $s$. This is of course related to the question how sharp an upper bound our method can possibly give.

[Lower bounds] A general, unconditional (and trivial) lower bound is:

\section{Proposition 3}

$$
\gamma_{K}>-\alpha_{K}-\beta_{K}-c_{K}
$$

This follows immediately from

$$
\gamma_{K}+\alpha_{K}+\beta_{K}+c_{K}=\sum_{\rho} \frac{1}{\rho}=\frac{1}{2} \sum_{\rho}\left(\frac{1}{\rho}+\frac{1}{\bar{\rho}}\right)>0
$$


(Under $(\mathrm{GRH})$, one can deduce this also from Proposition 2 (ii) by letting $x \rightarrow 1$.)

We note that the absolute value of the negative lower bound given above is much larger than that of the upper bound given in Theorem 1. For example, in the NF-case, the former is $\sim \frac{1}{2} \log |d|$ while the latter is $\sim 2 \log \log |d|$. This is not just because we have not assumed GRH for the above lower bound as we did for the upper bound. Indeed, in the FF-case, $\gamma_{K}$ can be of the order of

$$
\left(-\frac{1}{\sqrt{q}+1}+\varepsilon\right)\left(g_{K}-1\right) \log q
$$

for Shimura curves over $\mathbb{F}_{q}\left(q\right.$ : a square, $\left.g_{K} \rightarrow \infty\right)$; see $\S 2.1$. But we can show

Theorem 2 (FF) Fix q. Then

$$
\liminf \frac{\gamma_{K}}{\left(g_{K}-1\right) \log q} \geq-\frac{1}{\sqrt{q}+1}
$$

Proof This is obtained by using Corollary 1 of Tsfasman [ $\left.\mathrm{Ts}_{1}\right]$. (If we combine the inequality $h . m \leq a . m$ in (1.4.5) with the Drinfeld-Vladut asymptotic upper bound [D-V] for $N_{1}$, then what we obtain is a somewhat weaker statement, where the denominator $\sqrt{q}+1$ on the RHS of (1.6.23) is replaced by $\sqrt{q}$.

First, let us recall the basic materials from $\left[\mathrm{Ts}_{1}\right]$ that will be needed. By a curve over $\mathbb{F}_{q}$, we shall always mean a complete, smooth, geometrically irreducible algebraic curve over $\mathbb{F}_{q}$. For a curve $C$ over $\mathbb{F}_{q}$, let $g=g(C)$ denote the genus, and $B_{m}=B_{m}(C)$ denote the number of prime divisors (i.e., scheme theoretic closed points) of $C$ with degree $m$ over $\mathbb{F}_{q}$. Thus,

$$
N_{m}(C)=\sum_{d \mid m} d B_{d}(C)
$$

is the number of $\mathbb{F}_{q^{m}}$-rational points of $C$. If $\left\{C_{\alpha}\right\}$ is a family of curves over $\mathbb{F}_{q}$ ( $q$ : fixed) with growing genus such that

$$
\beta_{m}=\lim _{\alpha} \frac{B_{m}\left(C_{\alpha}\right)}{g\left(C_{\alpha}\right)}
$$

exists for all $m \geq 1$, we call the family $\left\{C_{\alpha}\right\}$ asymptotically exact. Each sequence of curves over $\mathbb{F}_{q}$ with growing genus contains a subsequence which is asymptotically exact $\left(\left[\mathrm{Ts}_{1}\right]\right.$ p.182). Moreover, for any asymptotically exact family of curves over $\mathbb{F}_{q}$, one has

$$
\sum_{m=1}^{\infty} \frac{m \beta_{m}}{q^{m / 2}-1} \leq 1
$$

(Corollary 1 in $\left.\left[\mathrm{Ts}_{1}\right]\right)$. 
Now, to prove Theorem 2, let us write $\gamma(C)=\gamma_{K}$, where $K$ is the function field of $C$. Put

$$
\lambda=\liminf \frac{\gamma(C)}{(g(C)-1) \log q}=\liminf \frac{\gamma(C)}{g(C) \log q} .
$$

Then there exists a family of curves $C$ over $\mathbb{F}_{q}$ with $g(C) \rightarrow \infty$ such that

$$
\lim _{C} \frac{\gamma(C)}{g(C) \log q}=\lambda
$$

and we may assume that this family is asymptotically exact. Let $C$ run over such a family. Then, by (1.4.3),

$$
\frac{\gamma(C)}{g(C) \log q}=\sum_{m=1}^{\infty} \frac{q^{m}+1-N_{m}(C)}{q^{m} g(C)}+\frac{1}{g(C)}\left(1-\frac{c_{q}}{\log q}\right) .
$$

Since the summand on the right-hand side of (1.6.25) has absolute value at most equal to $2 q^{-m / 2}$, the sum is uniformly convergent w.r.t. $C$. We thus obtain

$$
\begin{aligned}
\lambda & =\sum_{m=1}^{\infty} \lim _{C}\left(\frac{q^{m}+1-N_{m}(C)}{q^{m} g(C)}\right) \\
& =-\sum_{m=1}^{\infty} q^{-m}\left(\sum_{d \mid m} d \beta_{d}\right)=-\sum_{d=1}^{\infty} \frac{d \beta_{d}}{q^{d}-1} \\
& \geq-\frac{1}{\sqrt{q}+1}\left(\sum_{d=1}^{\infty} \frac{d \beta_{d}}{q^{d / 2}-1}\right) \geq-\frac{1}{\sqrt{q}+1}
\end{aligned}
$$

by (1.6.24), as desired.

Remark The above proof shows also that the equality $\lambda=-\frac{1}{\sqrt{q}+1}$ holds if and only if $\beta_{1}=\sqrt{q}-1$ holds, i.e., if and only if the Drinfeld-Vladut asymptotic upper bound for $\frac{N_{1}(C)}{g(C)}$ is attained by this family.

In $\S 2.1$, we shall show that when $q$ is a square, then the equality holds for (1.6.23) (see $(2.1 .11))$.

It is also a very interesting problem to find out the precise value of the quantity

$$
C=\liminf \frac{\gamma_{K}}{\alpha_{K}}
$$

in the NF-case. As for this, what the author obtained are : 
(i) By Proposition 3(§1.6), we have

$$
C \geq-1 \quad \text { (unconditionally). }
$$

(ii) If there exists an infinite unramified Galois extension $M / k$ over a number field $k$ in which some prime ideals $\mathfrak{p}_{1}, \cdots \mathfrak{p}_{m}$ of $k$ decompose completely, then, under (GRH), we can show easily that

$$
C \leq-\frac{1}{\alpha_{k}}\left(\sum_{i=1}^{m} \frac{\log N\left(\mathfrak{p}_{i}\right)}{N\left(\mathfrak{p}_{i}\right)-1}\right),
$$

by applying Theorem 1 (the first inequality in (1.6.8)) to finite intermediate extensions of $M / k$. For example, one may choose the examples given in Cor $9.3 \sim 9.5$ of $[\mathrm{T}-\mathrm{V}]$, among which (Cor 9.5 is an old example due to the present author, but) Cor 9.4 gives the best result. In this case,

$$
k=\mathbb{Q}(\sqrt{d}), \quad d=-d_{1} \times 73 \times 79,
$$

where $d_{1}$ is the product of all prime numbers $q$ with $13 \leq q \leq 61$. It has an infinite 2classfield tower in which ten primes above $2,3,5,7,11$ split completely. By taking $\mathfrak{p}_{1}, \cdots \mathfrak{p}_{m}$ to be these ten primes of $k$, we obtain by (1.6.29),

$$
C \leq-0.16352 \cdots \quad(\text { under GRH) }
$$

Now, after the present article was submitted to this Volume, Tsfasman has kindly informed me that he can prove better results, namely,

$$
-0.26049 \cdots \leq C \leq-0.17849 \cdots
$$

(the LHS inequality under GRH), by using $[\mathrm{T}-\mathrm{V}]$ for the LHS inequality, and an uncondi-

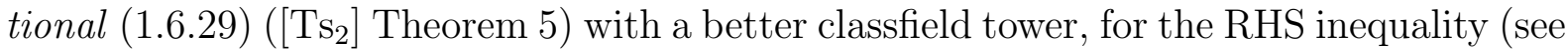
$\left.\left[\mathrm{Ts}_{2}\right]\right)$. The LHS inequality was surprising to the author who had considered it plausible that $C=-1 / 2$ (the value obtained by putting $q=1$ on the RHS of $(1.6 .23)$ ).

On the other hand,

$$
\limsup \frac{\gamma_{K}}{\alpha_{K}}=0 \quad \text { (under GRH) }
$$

by Theorems 1 , and 3 below (for, say, $N=2$ ).

When the degree of $K$ over $\mathbb{Q}(\mathrm{NF})$, or over a rational subfield of $K(\mathrm{FF})$, is relatively small, there is a much better lower bound for $\gamma_{K}$, as follows. 
Theorem 3 Put $k=\mathbb{Q}(\mathrm{NF}),=\mathbb{F}_{q}(t)(\mathrm{FF})$, and let $K$ be an extension of $k$ of degree $N>1$. Put

$$
\begin{aligned}
\alpha_{K}^{*}=\alpha_{K} /(N-1) & =\frac{\log \sqrt{|d|}}{N-1}(N F), \\
& =\frac{(g-1) \log q}{N-1}(F F),
\end{aligned}
$$

and assume $\alpha_{K}^{*}>1$. Then

$$
\begin{array}{rll}
\frac{\alpha_{K}^{*}+1}{\alpha_{K}^{*}-1}\left(\gamma_{K}+c_{K}\right) & >-2(N-1)\left(\log \alpha_{K}^{*}+1\right) & (N F, \text { under } G R H) \\
& >-2(N-1)\left(\log \alpha_{K}^{*}+\frac{\alpha_{K}^{*}}{\alpha_{K}^{*}-1}\right) & (F F) .
\end{array}
$$

Remarks (i) Granville-Stark $[\mathrm{G}-\mathrm{S}](\S 3.1)$ gave an equivalent statement when $[K: \mathbb{Q}]=$ 2 (in fact, $\left.L^{\prime}(1, \chi) / L(1, \chi)=\gamma_{K}-\gamma_{\mathbb{Q}}\right)$, whose argument applies also to abelian extensions over $\mathbb{Q}$. Our Theorem was motivated by $[\mathrm{G}-\mathrm{S}]$.

(ii) The bound given by Theorem 3 is sharp in the following sense. The RHS of (1.6.34) cannot be replaced by its quotient by such an $f(N)$ (NF) (resp. $f_{q}(N)$ (FF, for a fixed $q>2)$ ) that tends to $\infty$ as $N \rightarrow \infty$. This can be proved easily by using a family of $K$ satisfying (1.6.29) $(\mathrm{NF})$ resp. $(2.1 .8)(\S 2.1)(\mathrm{FF})$. The point is that, in each case, one can find a subsequence of $K$ such that $\alpha_{K} \rightarrow \infty$ and that the following (finite) limits $\lim \alpha_{K}^{*}>1$ and $\lim \frac{\gamma_{K}}{\alpha_{K}}<0$ exist.

Proof This will be based on the Main lemma and the following 4 inequalities;

$$
\begin{aligned}
\Phi_{K}(x) & \leq N \cdot \Phi_{k}(x) \\
\Phi_{k}(x) & <\log x \\
\ell_{K}(x) & \geq 0
\end{aligned}
$$

each for all $x>1$, and

$$
\beta_{K}<-[K: \mathbb{Q}]
$$

Among them, (1.6.35) is trivial, (1.6.36) $(\mathrm{FF})$ and (1.6.37) both follow directly from lemma 2 of $\S 1.5$., and (1.6.38) is because $\gamma+\log 2 \pi=2.415 \cdots>2$. The inequality $\Phi_{\mathbb{Q}}(x)<\log x$ can be proved easily as follows. Since $\Phi_{\mathbb{Q}}(x), \log x$ are both monotone increasing, it is enough to show $\Phi_{\mathbb{Q}}(x) \leq \log (x-1)$ for integers $x=n \geq 2$ (note the shift $x \rightarrow x-1$ on the right side). But by the prime factorization of $n$ !, we have

$$
\begin{aligned}
\log n !=\sum_{p^{k} \leq n}\left[\frac{n}{p^{k}}\right] \log p & \geq \sum_{p^{k} \leq n}\left(\frac{n+1}{p^{k}}-1\right) \log p \\
& =n \Phi_{\mathbb{Q}}(n+1),
\end{aligned}
$$


hence

$$
\Phi_{\mathbb{Q}}(n+1) \leq \frac{1}{n} \log (n !) \leq \log n
$$

for all $n \geq 1$.

Now we proceed to the proof of Theorem 3. By (1.6.35), (1.6.36), and then by (1.4.1), (1.5.2), (1.6.37), we obtain

$$
\begin{aligned}
N \log x & >\Phi_{K}(x)=\log x+\alpha_{K}+\beta_{K}+r_{K}(x)+\ell_{K}(x) \\
& \geq \log x+\alpha_{K}+\beta_{K}-\left(\frac{\sqrt{x}+1}{\sqrt{x}-1}\right)\left(\gamma_{K}+\alpha_{K}+\beta_{K}+c_{K}\right) .
\end{aligned}
$$

Therefore,

$$
(N-1) \log x>-\frac{2}{\sqrt{x}-1}\left(\alpha_{K}+\beta_{K}\right)-\frac{\sqrt{x}+1}{\sqrt{x}-1}\left(\gamma_{K}+c_{K}\right) .
$$

Putting $x=\left(\alpha_{K}^{*}\right)^{2}$, we obtain

$$
\frac{\alpha_{K}^{*}+1}{\alpha_{K}^{*}-1}\left(\gamma_{K}+c_{K}\right)>-2(N-1) \log \alpha_{K}^{*}-\frac{2}{\alpha_{K}^{*}-1}\left(\alpha_{K}+\beta_{K}\right) .
$$

The rest follows directly, by (1.6.38) in the NF-case.

Corollary 1 If $N$ and $q$ (in the FF-case) are fixed and $\alpha_{K}^{*} \rightarrow \infty$, then

$$
\begin{array}{rll}
\gamma_{K} & >-2(N-1+\varepsilon) \log (\log |d|) & \\
& >-2(N F, \text { under } G R H) \\
& &
\end{array}
$$

\subsection{Supplementary remarks related to computations of $\gamma_{K}$}

An accurate computation of $\gamma_{K}$ for each individual $K$ is not the main issue of this paper. Still, it should probably be pointed out that there are other ways of computing $\gamma_{K}$ that are better at least microscopically.

One is the classical Landau formula generalizing the well-known Euler formula for $K=\mathbb{Q}$. Let $K$ be a number field, let $\zeta_{K}(s)=\sum_{n=1}^{\infty} a_{n} n^{-s}$ be the Dirichlet series expansion of $\zeta_{K}(s)$ on $\operatorname{Re}(s)>1$, and put $S_{K}(x)=\sum_{n \leq x} a_{n}$, so that

$$
\zeta_{K}(s)=s \int_{1}^{\infty} S_{K}(x) x^{-s-1} d x \quad(\operatorname{Re}(s)>1) .
$$

Further, put $\kappa_{K}=\lim _{s \rightarrow 1}(s-1) \zeta_{K}(s)=\lim _{x \rightarrow \infty}\left(S_{K}(x) / x\right)$, and

$$
B_{K}(x)=\kappa_{K}^{-1} \sum_{n \leq x} a_{n} n^{-1}-\log x \quad(x \geq 1) .
$$


Then the Landau formula asserts that

$$
\gamma_{K}=\lim _{x \rightarrow \infty} B_{K}(x)
$$

(Compare this with (1.6.1), and note that $\log (x)$ appears with opposite signs !)

Now, there exist positive constants $\epsilon, C$ and $x_{0} \geq 1$ (all depending on $K$ ) such that

$$
\left|S_{K}(x)-\kappa_{K} x\right| \leq C x^{1-\epsilon} \quad\left(x \geq x_{0}\right) .
$$

So, we can change the order of integration and passage to the limit $s \rightarrow 1$, on the RHS of (1.7.1) with $S_{K}(x)-\kappa_{K} x$ in place of $S_{K}(x)$. This gives an expression of $\gamma_{K}$ in terms of the definite integral of $\left(S_{K}(x)-\kappa_{K} x\right) x^{-2} d x$ from 1 to $\infty$. But since that from 1 to $x$ is nothing but $\kappa_{K} B_{K}(x)-S_{K}(x) x^{-1}$, we obtain directly

$$
\left|\gamma_{K}-B_{K}(x)\right| \leq \kappa_{K}^{-1} C\left(1+\epsilon^{-1}\right) x^{-\epsilon} \quad\left(x \geq x_{0}\right) .
$$

As for (1.7.4), although certainly not the best possible bound (as the well-known case of $K=\mathbb{Q}(\sqrt{-1})$...counting lattice points in circles...indicates), a general method (cf.e.g.[La] VI) shows that one can take $\epsilon=[K: \mathbb{Q}]^{-1}$ and can compute $C$ by using geometry of numbers. For $[K: \mathbb{Q}]=2$, the exponent $=-1 / 2$ of $x$ in (1.7.5) is as strong as our conditional estimate given in $§ 1.6$. The constant $C$ computed by following the method of loc.cit. is generally large compared with $\alpha_{K}$, but the actual convergence seems considerably faster than what we expect from such a bound.

The second is for the case of quadratic fields. We have to rely on the notation of $\S 2.2$ below. When $K$ is imaginary, we have the Kronecker formula (2.2.1). When $K$ is real, there is also Hecke's formula [H], which gives

$$
\gamma_{K}=-\frac{1}{2} \log \left(d_{K}\right)+2 \gamma_{\mathbb{Q}}+\frac{1}{h_{K}} \sum_{C} i_{C} .
$$

Here, $C$ runs over the narrow ideal classes of $K, h_{K}$ is the narrow class number, and $i_{C}$ is defined as follows. Pick any ideal from $C^{-1}$ with a $\mathbb{Z}$-basis $\alpha_{1}, \alpha_{2}$ satisfying $\alpha_{1} \alpha_{2}^{\prime}-\alpha_{1}^{\prime} \alpha_{2}$ $>0\left(\alpha_{i}^{\prime}\right.$ is the conjugate of $\left.\alpha_{i}\right)$, and put

$$
\omega(y)=\frac{\alpha_{2} y+i \alpha_{2}^{\prime}}{\alpha_{1} y+i \alpha_{1}^{\prime}} \quad(i=\sqrt{-1}, \quad 0<y<\infty)
$$

(its image is a semi-circle in the complex upper half plane vertical to the real axis).Then

$$
i_{C}=-\frac{1}{\log \epsilon} \int_{\epsilon^{-1}}^{\epsilon} \log \left(|\eta(\omega)|^{2} \operatorname{Im}(\omega)^{1 / 2}\right) d y / y,
$$

where $\epsilon$ is the fundamental unit $>1$ of $K$. (There is a small error in $[\mathrm{H}]$. The formula for $m$ (2 lines below (4) of p.202) is actually that for $4 m$; the formulas in p. 203 will give $1 / 2$ of the residue and of the constant term, if $\log 4$ in (5) is replaced by $\log 2$.) 
For example, when $K=\mathbb{Q}(\sqrt{10})$, where $\epsilon=3+\sqrt{10}$ and $h=2$, (1.7.6) (rewritten as the average of $\left.-\frac{1}{2} \log \left(d_{K}\right)+2 \gamma_{\mathbb{Q}}+i_{C}\right)$ gives

$$
\gamma_{K}=\frac{1}{2}(0.868877+0.402405)=0.635641 .
$$

On the other hand, $B_{K}\left(10^{5}\right)=0.635861$, and the GRH bounds given by Proposition 2 of $\S 1.6$ for $x=10^{5}$ give $0.634696<\gamma_{K}<0.639418$. Finally, $A_{K}\left(10^{5}\right)=0.636813$, for

$$
A_{K}(x)=\log (x)-\Phi_{K}(x)-1 .
$$

\section{Part 2 Some special families of $K$}

\subsection{Curves over $\mathbb{F}_{q}$ with many rational points}

When $K$ corresponds to such a curve, $\gamma_{K}$ tends to be negative with a large absolute value. In fact, as a direct application of Theorem 1 , we obtain

Theorem 4 (FF) Fix any prime power $q$ and $\varepsilon>0$. Then

$$
\frac{\gamma_{K}}{\left(g_{K}-1\right) \log q}<\varepsilon-\frac{N_{1}(K)}{(q-1)\left(g_{K}-1\right)}
$$

holds as long as the exact constant field of $K$ is $\mathbb{F}_{q}$ and the genus $g_{K}$ of $K$ is sufficiently large. Here, $N_{1}(K)$ is the number of $\mathbb{F}_{q}$-rational points of the curve corresponding to $K$.

Proof Let $\alpha_{K}=\left(g_{K}-1\right) \log q>0$. Then Theorem 1 gives

$$
\gamma_{K}<\left(\frac{\alpha_{K}+1}{\alpha_{K}-1}\right)\left(2 \log \alpha_{K}+1+\log q-\Phi_{K}\left(\alpha_{K}^{2}\right)\right) .
$$

Let $g_{K}$ be so large that $\alpha_{K}>q$, and take $m \in \mathbb{N}$ such that

$$
q^{2} \leq q^{m} \leq \alpha_{K}^{2}<q^{m+1}
$$

Then, by the definition of $\Phi_{K}(x)$,

$$
\Phi_{K}\left(\alpha_{K}^{2}\right) \geq \frac{N_{1}(K)}{\alpha_{K}^{2}-1}\left(\alpha_{K}^{2}\left(\frac{1}{q}+\cdots+\frac{1}{q^{m}}\right)-m\right) \log q
$$


hence

$$
-\left(\frac{\alpha_{K}+1}{\alpha_{K}-1}\right) \Phi_{K}\left(\alpha_{K}^{2}\right) \leq-\frac{N_{1}(K)}{\left(\alpha_{K}-1\right)^{2}}\left\{\frac{\alpha_{K}^{2}\left(1-q^{-m}\right)}{q-1}-m\right\} \log q .
$$

Now let $g_{K}$ be so large that

$$
\alpha_{K}>(q-1)\left(\frac{\log \alpha_{K}}{\log q}+\frac{1}{2}\right)+1
$$

hence

$$
\alpha_{K}>\frac{1}{2}(q-1)(m+1)+1
$$

Then by (2.1.3), (2.1.4), we obtain $\alpha_{K}^{2} q^{-m}+m q<(m+1) q<2 \alpha_{K}+m-1$; hence

$$
\alpha_{K}^{2}\left(1-q^{-m}\right)-m(q-1)>\left(\alpha_{K}-1\right)^{2} .
$$

Therefore,

$$
-\left(\frac{\alpha_{K}+1}{\alpha_{K}-1}\right) \Phi_{K}\left(\alpha_{K}^{2}\right)<-\frac{N_{1}(K)}{q-1} \log q
$$

hence by (2.1.2), we obtain

$$
\frac{\gamma_{K}}{\alpha_{K}}<\left(\frac{\alpha_{K}+1}{\alpha_{K}-1}\right)\left(\frac{2 \log \alpha_{K}}{\alpha_{K}}+\frac{1+\log q}{\alpha_{K}}\right)-\frac{N_{1}(K)}{(q-1)\left(g_{K}-1\right)} .
$$

Therefore, if $g_{K}$ is so large that the first term on the RHS is $<\varepsilon$, we have

$$
\frac{\gamma_{K}}{\alpha_{K}}<\varepsilon-\frac{N_{1}(K)}{(q-1)\left(g_{K}-1\right)} .
$$

We shall combine this with two typical results on curves with many rational points. First, we refer to

Theorem (Elkies-Howe-Kresch-Poonen-Wetherell-Zieve)[E-](§3.2) There exists a positive absolute constant $c_{0}$ such that for any prime power $q$, and any $g \geq 1$, there exists $a$ curve $X$ over $\mathbb{F}_{q}$ with genus $g$ such that

$$
\# X\left(\mathbb{F}_{q}\right) \geq c_{0}(\log q)(g-1)
$$


Combining this with Theorem 4, we obtain

Corollary 1 For any fixed prime power $q$, we have

$$
C(q) \leq-c_{0} \frac{\log q}{q-1}
$$

Corollary 2 Fix any prime power $q$. Then, for any sufficiently large $g$, there exists $K$ over $\mathbb{F}_{q}$ with genus $g$ such that $\gamma_{K}<0$.

Secondly, let us recall

Theorem $\left(\left[\mathrm{I}_{1}\right] \sim\left[\mathrm{I}_{2}\right],[\mathrm{TVZ}]\right)$ When $q$ is a square, there exist Shimura curves $X$ over $\mathbb{F}_{q}$ with growing genus $g$ such that

$$
\# X\left(\mathbb{F}_{q}\right) \geq(\sqrt{q}-1)(g-1) .
$$

Therefore, by Theorems 2 and 4, we obtain

Corollary 1 Let $q$ be a square. Then

$$
C(q)=-\frac{1}{\sqrt{q}+1}
$$

Corollary 2 Let $q$ be a square, and $K$ be the function field of a Shimura curve over $\mathbb{F}_{q}$ corresponding to a $(\infty \times p)$-adic discrete subgroup $\Gamma$ in the sense of $\left[\mathrm{I}_{1}\right] \sim\left[\mathrm{I}_{2}\right]$. Suppose that $\Gamma$ is torsion-free, and

$$
\begin{cases}\sqrt{q}>3 \\ g-1>3(q+1) / 2(\sqrt{q}-3)\end{cases}
$$

Then $\gamma_{K}<0$.

Proof In Proposition 2(i), take $x=q^{2}$. Then

$$
\begin{aligned}
\gamma_{K} & \leq \frac{q+1}{q-1}\left(2 \log q-\Phi_{K}\left(q^{2}\right)\right)+\frac{2 \alpha_{K}}{q-1}-c_{q} \\
& \leq \frac{q+1}{q-1}\left(2 \log q-\frac{N_{1}(K)}{q^{2}-1}(q-1) \log q\right)+\frac{2 \alpha_{K}}{q-1}-c_{q} .
\end{aligned}
$$

But $N_{1}(K) \geq(\sqrt{q}-1)\left(g_{K}-1\right)$ when $K$ corresponds to such a Shimura curve. Therefore,

$$
\begin{aligned}
\frac{\gamma_{K}}{\alpha_{K}} & \leq \frac{q+1}{q-1}\left(\frac{2}{g_{K}-1}-\frac{\sqrt{q}-1}{q+1}\right)+\frac{2}{q-1}-\frac{c_{q}}{\alpha_{K}} \\
& =\frac{1}{q-1}\left\{(3-\sqrt{q})+\frac{3}{2} \frac{q+1}{g_{K}-1}\right\},
\end{aligned}
$$

from which the desired assertion follows at once. 


\subsection{Imaginary quadratic fields}

Let $K$ be an imaginary quadratic field with discriminant $d(<0)$. Then the Kronecker limit formula, averaged over all the ideal classes of $K$, gives

\section{Theorem (Kronecker)}

$$
\gamma_{K}=-\frac{1}{2} \log |d|+2 \gamma_{\mathbb{Q}}-\log 2+\frac{1}{h} \sum_{C} t_{C}
$$

Here, $h=h_{K}$ is the class number of $K, C$ runs over all ideal classes of $K$,

$$
\begin{gathered}
t_{C}=-2 \log \left(\left|\eta\left(\omega_{C}\right)\right|^{2} \cdot \operatorname{Im}\left(\omega_{C}\right)^{1 / 2}\right), \\
\eta(\tau)=q^{\frac{1}{24}} \prod_{n=1}^{\infty}\left(1-q^{n}\right), \quad q=e^{2 \pi i \tau} \quad(\operatorname{Im}(\tau)>0)
\end{gathered}
$$

is the Dedekind $\eta$-function, and $\omega_{C}$ is defined as follows. Pick any ideal from $C^{-1}$, with such a $\mathbb{Z}$-basis $\left[\omega_{1}, \omega_{2}\right]$ that $\operatorname{Im}\left(\omega_{2} / \omega_{1}\right)>0$. Then we put $\omega_{C}=\omega_{2} / \omega_{1}$. Since the function

$$
|\eta(\tau)|^{2}(\operatorname{Im}(\tau))^{\frac{1}{2}}
$$

is $\mathrm{SL}_{2}(\mathbb{Z})$-invariant, $t_{C}$ is well-defined.

Remark Each $t_{C}$ is positive. Indeed, the maximal value $M$ of (2.2.4) on the upper half plane is attained at $\tau=\frac{1}{2}(1+\sqrt{3} i)$, and

$$
M=0.596450134 \cdots, \quad \log M=-0.516759638 \cdots .
$$

Since $-t_{C} \leq 2 \log M<0$, we see that $t_{C}$ is always positive.

Now, one notes that the contribution of the principal ideal class $C_{0}$ to the formula (2.2.1) is extraordinarily large. Indeed

$$
t_{C_{0}} \sim \frac{1}{6} \pi \sqrt{|d|}
$$

which is "too large" compared with our upper bound $\sim \log \log |d|$ for $\gamma_{K}$ under GRH. So, this "too outstanding a contribution of the principal class" should be "pulled down" by averaging over a large number of non-principal classes. We thus obtain, by combining with Theorem 1, the following 
Theorem 5(under $(\mathrm{GRH}))$. If $\alpha_{K}=\frac{1}{2} \log \left|d_{K}\right|>1.16$. (i.e., $\left|d_{K}\right| \geq 11$ ), then

$$
h_{K}>\frac{\frac{\pi}{6} \sqrt{\left|d_{K}\right|}-\alpha_{K}+b_{1}}{\alpha_{K}+2 \log \alpha_{K}+b_{2}+c\left(\alpha_{K}\right)},
$$

with small $b_{1}, b_{2}, c\left(\alpha_{K}\right)$ given by

$$
\left\{\begin{array}{l}
b_{1}=2 \log M+\log 2-4 q_{0}=-0.34037-4 q_{0}, \quad q_{0}=e^{-\pi \sqrt{\left|d_{K}\right|}} \\
b_{2}=2 \log M-2 \gamma_{\mathbb{Q}}+\log 2+1=-0.49480 \cdots \\
c(t)=\frac{4 \log t+2}{t-1}
\end{array}\right.
$$

Proof Write

$$
\left\{\begin{array}{l}
\gamma_{K}=\frac{1}{h}\left(\sum_{C} t_{C}\right)-\xi \\
\xi=\alpha_{K}-2 \gamma_{\mathbb{Q}}+\log 2
\end{array}\right.
$$

Take $t_{0}>t_{1}>0$ such that

$$
t_{0} \leq t_{C_{0}}, \quad t_{1} \leq t_{C} \quad(\text { all } C)
$$

and a majorant $U$ for $\gamma_{K}$;

$$
\gamma_{K} \leq U
$$

Then

$$
\frac{t_{0}-t_{1}}{h}+t_{1} \leq \frac{1}{h}\left(\sum_{C} t_{C}\right) \leq U+\xi
$$

hence $U+\xi-t_{1}$ is positive and

$$
h \geq \frac{t_{0}-t_{1}}{U+\xi-t_{1}} .
$$

For $t_{1}$, we choose $t_{1}=-2 \log M$; and for $t_{0}$, we may choose

$$
t_{0}=\frac{\pi \sqrt{\left|d_{K}\right|}}{6}-4 q_{0}-\log \frac{\sqrt{\left|d_{K}\right|}}{2} \quad\left(q_{0}=e^{-\pi \sqrt{\left|d_{K}\right|}}\right)
$$

(see below). Finally, choose $U$ as in Theorem 1 ,

$$
U=\left(\frac{\alpha_{K}+1}{\alpha_{K}-1}\right)\left(2 \log \alpha_{K}+1\right)
$$


and we obtain the Theorem by (2.2.13). Here, it remains to check that $t_{0}$ given by $(2.2 .14)$ satisfies both

$$
\text { (i) } t_{0} \leq t_{C_{0}} \text { and (ii) }-2 \log M<t_{0} \text {. }
$$

(i) For $\omega_{C_{0}}$, we may choose $\left(\sqrt{\left|d_{K}\right|} i\right) / 2$, resp. $\left(1+\sqrt{\left|d_{K}\right|} i\right) / 2$, according to whether $d_{K} \equiv 0(\bmod 4)$, resp. $\equiv 1(\bmod 4) ;$ hence

$$
t_{C_{0}}=-\log \frac{\sqrt{\left|d_{K}\right|}}{2}+\frac{\pi \sqrt{\left|d_{K}\right|}}{6}-4 \log \prod_{n=1}^{\infty}\left(1-\left(\varepsilon q_{0}\right)^{n}\right),
$$

with $\varepsilon=1$ resp. -1 . But $\prod_{n=1}^{\infty}\left(1-\left(\varepsilon q_{0}\right)^{n}\right)<1$ resp. $<1+q_{0}$; hence

$$
t_{C_{0}}>-\log \frac{\sqrt{\left|d_{K}\right|}}{2}+\frac{\pi \sqrt{\left|d_{K}\right|}}{6}-\left\{\begin{array}{llll}
0 & \cdots & d_{K} \equiv 0 & (\bmod 4), \\
4 q_{0} & \cdots & d_{K} \equiv 1 & (\bmod 4),
\end{array}\right.
$$

which settles (i). (ii) For $\alpha_{K}>1.16$, we have $t_{0}>1.2032>1.0335 \ldots=-2 \log M$.

Remark As the above proof shows, the term $-4 q_{0}$ in the formula for $b_{1}$ in Theorem 5 is unnecessary when $d_{K} \equiv 0(\bmod 4)$.

A similar result has already been obtained by Granville-Stark[G-S] (Th 1; note that the unconditional Th 2 still contains $\left.L^{\prime}(1, \chi) / L(1, \chi)=\gamma_{K}-\gamma_{\mathbb{Q}}\right)$.

S.Louboutin kindly informed the author that, as an asymptotic formula, there is an essentially stronger (and much older) result due to Littlewood [Li];

$$
\frac{h \log \log |d|}{\sqrt{|d|}}>\frac{\pi \cdot \exp \left(-\gamma_{\mathbb{Q}}\right)}{12}-o(1) \quad \text { (under GRH). }
$$

(As asymptotic formula, this is better than Theorem 5 when $\log |d| / \log \log |d| \geq 4 \exp \left(\gamma_{\mathbb{Q}}\right)$; hence when $|d|$ has 10 or more digits.)

\subsection{The field $K_{p}$}

For each odd prime $p$, let $K_{p}$ denote the unique cyclic extension of degree $p$ over $\mathbb{Q}$ contained in the field $\mathbb{Q}\left(\boldsymbol{\mu}_{p^{2}}\right)$ of $p^{2}$-th roots of unity. It is totally real, with discriminant $d=d_{p}=p^{2 p-2} ;$ whence

$$
\log \sqrt{d}=(p-1) \log p
$$


Let $\ell$ be any prime number $\neq p$. Then, by classfield theory,

$$
\ell \text { decomposes completely in } K_{p} \longleftrightarrow \ell^{p-1} \equiv 1 \quad\left(\bmod p^{2}\right) .
$$

We shall study the invariant

$$
\gamma_{p}=\gamma_{K_{p}}
$$

in connection with the following set of primes

$$
W(p)=\left\{\ell ; \text { primes }<p, \quad \ell^{p-1} \equiv 1\left(\bmod p^{2}\right)\right\} .
$$

For example, the list of non-empty $W(p)$ with $p<100$ is

$$
\begin{array}{lll}
W(11)=\{3\}, & W(43)=\{19\}, & W(59)=\{53\}, \\
W(71)=\{11\}, & W(79)=\{31\}, & W(97)=\{53\} .
\end{array}
$$

Among the 14 primes $p$ with $900<p<1000$, only 3 primes $p$ satisfy $W(p) \neq \emptyset$ (namely, $p=907,919,983$ ). The known primes $p$ such that $W(p)$ contains 2 (resp. 3 ) are $p=1093,3511$ (resp. $p=11,1006003$ ).

Theorem 6 Under (GRH) for $K_{p}$, we have

(i).

$$
\gamma_{p}<i_{p}^{\prime}\{2 \log (p-1)+2 \log \log p+1\}-p\left(\sum_{\substack{\ell^{k}<p \\ \ell \in W(p)}} \frac{\log \ell}{\ell^{k}}\right)
$$

(ii).

$$
\gamma_{p}>-i_{p}\{2(p-1)(\log \log p+1)\}-1
$$

Here,

$$
i_{p}^{\prime}=1+\frac{2}{(p-1) \log p-1}, \quad i_{p}=1-\frac{2}{\log p+1} .
$$

The first inequality is a direct consequence of Theorem $1(\S 1.6)$. Indeed, each $\ell \in W(p)$ has $p$ distinct primes of $K_{p}$ above $\ell$, so if we write $\alpha_{p}=\alpha_{K_{p}}=(p-1) \log p$, then (since $\alpha_{p}^{2}-p \geq\left(\alpha_{p}-1\right)^{2}$ for $\left.p \geq 3\right)$ we have

$$
\frac{\alpha_{p}+1}{\alpha_{p}-1} \Phi_{K}\left(\alpha_{p}^{2}\right) \geq p \sum_{\substack{\ell^{k}<p \\ \ell \in W(p)}} \frac{\alpha_{p}^{2}-\ell^{k}}{\left(\alpha_{p}-1\right)^{2}} \frac{\log \ell}{\ell^{k}} \geq p \sum_{\substack{\ell^{k}<p \\ \ell \in W(p)}} \frac{\log \ell}{\ell^{k}} .
$$

The second inequality is a special case of Theorem 3 . Note that $\alpha_{K}^{*}=\log p$. 
Remark One may replace the sum

$$
\sum_{\substack{\ell^{k}<p \\ \ell \in W(p)}} \frac{\log \ell}{\ell^{k}}
$$

in Theorem 6(i) and the following Corollaries 1, 2, by a somewhat larger sum

$$
\sum_{\ell, k} \frac{\log \ell}{\ell^{k}}
$$

where the primes $\ell$ satisfy $\ell^{p-1} \equiv 1\left(\bmod p^{2}\right)$ and $k$ satisfies $\ell^{k}<2 \alpha_{p}-1$ (instead of $\left.\ell^{k}<p\right)$. The latter sum is also interesting, but in order not to blur the present focus, we just give it as a remark instead of incorporating it in Theorem 6.

Combining (i) and (ii) of Theorem 6, we obtain immediately:

Corollary 1 (under $(\mathrm{GRH})$ ) For any $\varepsilon>0$, there is an effectively computable bound $N_{\varepsilon}$ such that if $p>N_{\varepsilon}$ then

$$
\sum_{\substack{\ell^{k}<p \\ \ell \in W(p)}} \frac{\log \ell}{\ell^{k}}<2 \log \log p+2+\varepsilon .
$$

Since $\sum_{\ell^{k}<x}(\log \ell) / \ell^{k} \sim \log x$, this is in accordance with a result of Lenstra [Le](Th 3) which asserts (unconditionally!) that there exists some prime $\ell \notin W(p)$ with $\ell<4(\log p)^{2}$.

Remark Lenstra also gives an asymptotic bound

$$
\left(4 e^{-2}+\varepsilon\right)(\log p)^{2}
$$

The accordance would have been perfect, if the second term on the right side of (2.3.6) had been $\log 4-2$ instead of 2 . But we have not been able to make this replacement.

Corollary 2 (under $(\mathrm{GRH})$ )

(i).

$$
\lim \left(\frac{\gamma_{p}}{(p-1) \log p}\right)=0
$$

(ii).

$$
\limsup \left(\frac{\gamma_{p}}{p}\right) \leq \limsup \left(\frac{\gamma_{p}}{p}+\sum_{\substack{\ell^{k}<p \\ \ell \in W(p)}} \frac{\log \ell}{\ell^{k}}\right) \leq 0
$$

(iii).

$$
-2 \leq \liminf \left(\frac{\gamma_{p}}{p \log \log p}\right)
$$


By Theorem 6 (i), if $W(p)$ contains small primes $\ell$, then $\gamma_{p}$ tends to be negative. For example, $\gamma_{3}, \gamma_{5}, \gamma_{7}$ are positive, but $\gamma_{11}$ is negative, reflecting $W(11) \ni 3$. Also, $\gamma_{1093}$ is "very negative", reflecting $W(1093) \ni 2$ (see Table $(2.3 A)$ ). It is an interesting problem to investigate asymptotic behaviors of $\gamma_{p}, \gamma_{p} / p$, etc. In particular, the determination of the value of

$$
\liminf \frac{\gamma_{p}}{p} \quad(\leq 0)
$$

will have the following implications.

Corollary 3 (under $(\mathrm{GRH})$ )

(i). If $\lim \inf \frac{\gamma_{p}}{p}=0$, then, for each prime $\ell$, there exist at most finitely many primes $p$ that satisfy

$$
\ell^{p-1} \equiv 1 \quad\left(\bmod p^{2}\right)
$$

(ii). If, for each prime $\ell$, all but finitely many primes $p$ satisfy

$$
\ell^{p-1} \equiv 1 \quad\left(\bmod p^{2}\right)
$$

then $\liminf \frac{\gamma_{p}}{p}=-\infty$.

Proof (i) If for some $\ell$ there exist infinitely many $p$ such that $\ell^{p-1} \equiv 1\left(\bmod p^{2}\right)$, then by Theorem 6(i),

$$
\liminf \frac{\gamma_{p}}{p} \leq-\frac{\log \ell}{\ell-1}<0
$$

(ii) Under this assumption, by Theorem 6(i),

$$
\liminf \frac{\gamma_{p}}{p} \leq-\sum_{\ell} \frac{\log \ell}{\ell-1}=-\infty
$$

Table 2.3A shows the approximate values of $\gamma_{p}$ for $p<110$, and for several primes around $p=1093,3511$ (the two known $p$ such that $W(p)$ contains 2 ), under GRH. Let $\ell_{p}$ (resp. $u_{p}$ ) denote the lower (resp. upper) bound for $\gamma_{p}$ given by Proposition 2 (§1.6) for $x=x_{0}=5 \times 10^{4}$, and put

$$
\begin{aligned}
\gamma_{p}^{\prime} & =\frac{1}{2}\left(\ell_{p}+u_{p}\right), \\
\gamma_{p}^{\prime \prime} & =\log x_{0}-\Phi_{K_{p}}\left(x_{0}\right)-1, \\
\varepsilon_{p} & =\frac{1}{2}\left(u_{p}-\ell_{p}\right),
\end{aligned}
$$

so that (under GRH) $\gamma_{p}$ should lie in-between $\gamma_{p}^{\prime} \pm \varepsilon_{p}$, and $\gamma_{p}^{\prime \prime}$ should also be close to $\gamma_{p}$.

Note how "conspicuously negative" the values of $\gamma_{p}^{\prime}, \gamma_{p}^{\prime \prime}$ are when $W(p)$ contains small primes! 
Table 2.3A:

\begin{tabular}{|c|c|c|c|}
\hline $\mathrm{p}$ & $\gamma_{p}^{\prime \prime}$ & $\gamma_{p}^{\prime}$ & $\varepsilon_{p}$ \\
\hline 3 & 1.76673 & 1.76741 & 0.00270354 \\
\hline 5 & 1.6981 & 1.69927 & 0.0122214 \\
\hline 7 & 1.84553 & 1.84723 & 0.032591 \\
\hline 11 & -1.43302 & -1.43032 & 0.0577191 \\
\hline 13 & 0.468641 & 0.472016 & 0.107757 \\
\hline 17 & 3.5781 & 3.58283 & 0.210134 \\
\hline 19 & 4.53435 & 4.53974 & 0.25948 \\
\hline 23 & 4.47064 & 4.47731 & 0.346256 \\
\hline 29 & 2.32308 & 2.33163 & 0.46998 \\
\hline 31 & 4.61964 & 4.62896 & 0.540857 \\
\hline 37 & 5.6061 & 5.6175 & 0.70755 \\
\hline 41 & 4.2761 & 4.28883 & 0.805977 \\
\hline 43 & -0.929757 & -0.916538 & 0.81594 \\
\hline 47 & -2.6783 & -2.66375 & 0.91587 \\
\hline 53 & 6.05396 & 6.071 & 1.17309 \\
\hline 59 & 0.428977 & 0.447956 & 1.30809 \\
\hline 61 & 4.62301 & 4.64288 & 1.40864 \\
\hline 67 & 6.03706 & 6.05918 & 1.6139 \\
\hline 71 & -12.8724 & -12.8496 & 1.57591 \\
\hline 73 & 5.99832 & 6.02267 & 1.81104 \\
\hline 79 & -3.85765 & -3.83146 & 1.92486 \\
\hline 83 & 1.21387 & 1.24177 & 2.10718 \\
\hline 89 & 7.51911 & 7.54953 & 2.37227 \\
\hline 97 & -5.02725 & -4.99428 & 2.54395 \\
\hline 101 & 2.75934 & 2.79415 & 2.75782 \\
\hline 103 & -2.22423 & -2.18885 & 2.7859 \\
\hline 107 & 5.75378 & 5.79103 & 3.00361 \\
\hline 109 & 5.59505 & 5.63306 & 3.07587 \\
\hline 1069 & -4.10435 & -3.63507 & 51.7394 \\
\hline 1087 & -5.5176 & -5.03975 & 52.7617 \\
\hline 1091 & -3.11201 & -2.63214 & 53.0135 \\
\hline 1093 & -748.191 & -747.74 & 46.4644 \\
\hline 1097 & 3.54759 & 4.03061 & 53.4188 \\
\hline 1103 & 7.84455 & 8.33062 & 53.8033 \\
\hline 1109 & -0.666736 & -0.178118 & 54.0736 \\
\hline 3499 & 9.81761 & 11.521 & 206.78 \\
\hline 3511 & -2423.07 & -2421.45 & 185.836 \\
\hline 3517 & 7.66195 & 9.37476 & 207.986 \\
\hline
\end{tabular}




\subsection{The field index of $K_{p}$}

We shall give some applications to the field index of $K_{p}$ in the sense of [G]. In general, let $K$ be a number field and $O_{K}$ be the ring of integers of $K$. For each $\xi \in O_{K}$, consider the discriminant $D(\xi)=I(\xi)^{2} \cdot d_{K}$ of $\xi$. Thus, $I(\xi)=\left(O_{K}: \mathbb{Z}[\xi]\right)$. The greatest common divisor $I_{K}$ of $I(\xi)$ is called the field index of $K$. Clearly, $I_{K}=1$ if $O_{K}$ is generated by a single element. When $K=K_{p}$, write $I_{K}=I_{p}$. Then

$$
\ell \mid I_{p} \longleftrightarrow \ell \in W(p)=\left\{\ell ; \text { primes }<p, \quad \ell^{p-1} \equiv 1\left(\bmod p^{2}\right)\right\} .
$$

This is obvious by the Dirichlet pigeon hole principle ( $p$ pigeons are the conjugates of $\xi$, and $\ell$ holes are the residues classes modulo a fixed prime factor of $\ell$ ) and the Chinese remainder theorem. In particular, $I_{p}>1$ if and only if $W(p) \neq \emptyset$. The exponent of $\ell \in W(p)$ in $I_{p}$ can be expressed explicitly as

$$
\operatorname{ord}_{\ell} I_{p}=\sum_{\ell^{k}<p} C\left(p, \ell^{k}\right),
$$

where $C(m, n)$ denotes the following combinatorial number.

Consider finite sets $M, N$ of orders $m, n$, respectively, with $m>n$. For each map $f: M \rightarrow N$, let $n_{f}$ be the number of unordered pairs $\left(\mu, \mu^{\prime}\right)$ of distinct elements of $M$ such that $f(\mu)=f\left(\mu^{\prime}\right)$. Define $C(m, n)=\operatorname{Min}_{f}\left(n_{f}\right)(>0)$. Clearly, $n_{f}$ attains the minimal value $C(m, n)$ if and only if the maximal difference among $\# f^{-1}(\mu)(\mu \in N)$ is at most 1. (So, if we write $M=\{1,2, \cdots, m\}$, the $\bmod \ell^{k} \operatorname{map} f_{k}: M \rightarrow \mathbb{Z} / \ell^{k}$ satisfies $n_{f_{k}}=C\left(m, \ell^{k}\right)$ for all $k$ such that $\ell^{k}<m$. This explains the remaining key point underlying the equality (2.4.1).) Explicitly,

$$
C(m, n)=\left[\frac{m}{n}\right]\left(m-\frac{1}{2} n-\frac{1}{2}\left[\frac{m}{n}\right] n\right) .
$$

We have

$$
\frac{m}{2 n}(m-n) \leq C(m, n) \leq \frac{m}{2 n}(m-n)+\frac{n}{8} .
$$

(In fact, the left and the right sides of (2.4.3) are given respectively by

$$
C(m, n)-\frac{1}{2 n} k(n-k), \quad C(m, n)+\frac{1}{8 n}(n-2 k)^{2},
$$

where $k$ is defined by $m=\left[\frac{m}{n}\right] n+k(0 \leq k<n)$.) Since

$$
\begin{aligned}
& \log I_{p}=\sum_{\ell \in W(p)} \operatorname{ord}_{\ell}\left(I_{p}\right) \log \ell \\
& =\sum_{\substack{\ell \in W(p) \\
\ell^{k}<p}} C\left(p, \ell^{k}\right) \log \ell,
\end{aligned}
$$


we see that $\log I_{p}$ is fairly close to, and is bounded from below, by

$$
\frac{p}{2} \sum_{\substack{\ell \in W(p) \\ \ell^{k}<p}}\left(\frac{p}{\ell^{k}}-1\right) \log \ell=\frac{1}{2}(p-1) \Phi_{K_{p}}(p) .
$$

This was the initial motivation for our study of $\Phi_{K}(x)$. Combining with our previous bounds for $\gamma_{p}$, we obtain some estimations of $\log I_{p}$, as follows.

Proposition 4 (under $(\mathrm{GRH})$ )

(i). For each $\varepsilon>0$, if $p \geq N_{\varepsilon}$, then

$$
\log I_{p}<(1+\varepsilon) p^{2} \log \log p .
$$

(ii). If $\gamma_{p}<-2 \sqrt{p} \log p$, then $W(p) \neq \emptyset$, and

$$
\log I_{p}>\frac{1}{2}\left(\gamma_{\mathbb{Q}}+\log 4 \pi\right) p \sqrt{p}
$$

Sketch of proof (i) By (2.4.3) (2.4.4), we have

$$
\begin{aligned}
\log I_{p} & \leq \frac{p-1}{2} \Phi_{K_{p}}(p)+\frac{1}{8} \sum_{\substack{\ell \in W(p) \\
\ell^{k}<p}} \ell^{k} \log \ell \\
& <\frac{p-1}{2} \Phi_{K_{p}}(p)+\frac{p}{8}\left(\sum_{\ell^{k}<p} \log \ell\right) .
\end{aligned}
$$

By using the explicit formula for $\Phi_{K_{p}}(p)$, together with the upper bound

$$
r_{K_{p}}(p) \leq-\frac{\sqrt{p}-1}{\sqrt{p}+1}\left(\alpha_{K_{p}}+\beta_{K_{p}}+\gamma_{p}+1\right)
$$

(Main lemma and (1.3.11)), we obtain

$$
\log I_{p}<-\frac{p-1}{2} \gamma_{p}+O\left(p^{2}\right)
$$

Therefore, by Theorem 6(ii), we obtain

$$
\frac{\log I_{p}}{p^{2} \log \log p}<1+o(1)
$$

(ii) We have

$$
\log I_{p} \geq \frac{p-1}{2} \Phi_{K_{p}}(p) .
$$

By using the explicit formula for $\Phi_{K_{p}}(p)$, the lower bound for $r_{K_{p}}(p)((1.5 .2)(1.4 .1))$, and the inequality $\ell_{K_{p}}(p)>\log p$ (by (1.5.9)), we obtain

$$
\Phi_{K_{p}}(p)>-2 \sqrt{p} \log p+\sqrt{p}\left(\gamma_{\mathbb{Q}}+\log 4 \pi\right)-\frac{\sqrt{p}+1}{\sqrt{p}-1}\left(\gamma_{p}+1\right),
$$

from which the desired inequality follows directly. 


\subsection{The field index of $K_{p}$ (continued)}

Finally, we shall show that the above conditional upper bound (Proposition 4(i)) for $\log I_{p}$ is essentially stronger than what one can obtain by the "easier" method, i.e., by using the index of a standard generator of $K_{p}$. Put

$$
\left\{\begin{aligned}
\Delta_{p} & =\left\{\delta \in\left(\mathbb{Z} / p^{2}\right)^{\times} ; \delta^{p-1}=1\right\}, \\
\eta_{p} & =\sum_{\delta \in \Delta_{p}} \zeta^{\delta}
\end{aligned}\right.
$$

where $\zeta=\exp \left(2 \pi \sqrt{-1} / p^{2}\right)$. Then $K_{p}=\mathbb{Q}\left(\eta_{p}\right)$, and the ring of integers $O_{p}$ is spanned over $\mathbb{Z}$ by 1 and the conjugates of $\eta_{p}$. (The trace of $\eta_{p}$ is 0 ). As $I_{p}$ divides $I\left(\eta_{p}\right)$, any estimation of the latter from above gives rise to that of the former. Recall that

$$
D\left(\eta_{p}\right)=I\left(\eta_{p}\right)^{2} p^{2 p-2} .
$$

By Lemma 3 below, we obtain

$$
D\left(\eta_{p}\right)<p^{p} \cdot(p-1)^{p-1} \cdots \cdot 2^{2} \cdot 1^{1}
$$

This gives

$$
\begin{aligned}
\log D\left(\eta_{p}\right) & <\sum_{i=1}^{p} i(\log i) \\
& <\frac{1}{2} p^{2} \log p-\frac{1}{4} p^{2}+p \log p+1-2 \log 2
\end{aligned}
$$

and hence

Proposition 5 (unconditional)

$$
\log I_{p}<\left(\frac{1}{4}+\varepsilon\right) p^{2} \log p
$$

Note the difference "log log vs.log", between Proposition 4(i) and Proposition 5.

Remarks (i) Mahoro Shimura has shown by numerical computations that the upper bound for $\log D\left(\eta_{p}\right)$ provided by (2.5.3) is quite close to the actual values (the ratio of their $\log$ is $0.98 \cdots$ for $p \doteqdot 400)$.

(ii) Each prime factor $\ell$ of $I\left(\eta_{p}\right)$ must satisfy $\ell^{p-1} \equiv 1\left(\bmod p^{2}\right)$, but not necessarily $\ell<p$. Shimura has also shown that $I\left(\eta_{p}\right)$ is often divisible by much larger primes $\ell$. This phenomenon appears already at $p=11$, where $I_{11}=3^{17}$ but $I\left(\eta_{11}\right)=3^{22} \times 457$, and continues on. 
We conclude this section by stating the lemma in question. Take $n(\geq 2)$ real numbers $x_{1}, \ldots, x_{n}$, and put

$$
\left\{\begin{array}{l}
n S=x_{1}+\cdots+x_{n} \\
n T=x_{1}^{2}+\cdots+x_{n}^{2}
\end{array}\right.
$$

Then $S^{2} \leq T$, with the equality only if $x_{1}=\cdots=x_{n}$. Now fix $S, T \in \mathbb{R}$ satisfying $S^{2}<T$, and let $x_{1}, \ldots, x_{n}$ vary under the restrictions (2.5.6). Consider the maximal value of the discriminant

$$
D=\prod_{1 \leq i<j \leq n}\left(x_{i}-x_{j}\right)^{2}
$$

under (2.5.6).

Lemma 3 (Schur) The maximal value of $D$ is attained at the unique $n$-ple $\left(x_{1}^{0}, \cdots, x_{n}^{0}\right) \in$ $\mathbb{R}^{n}$ determined as follows. Put

$$
f(x)=\prod_{i=1}^{n}\left(x-x_{i}^{0}\right) .
$$

Then this monic polynomial of degree $n$ is determined uniquely by the differential equation

$$
f^{\prime \prime}(x)+(a-b x) f^{\prime}(x)+n b f(x)=0,
$$

where

$$
b=\frac{n-1}{T-S^{2}}, \quad a=b S
$$

The maximal value of $D$ is given by

$$
D=\frac{n^{n}(n-1)^{n-1} \cdots 2^{2} 1^{1}}{b^{\frac{1}{2} n(n-1)}} .
$$

Remark This "another Schur's lemma" was kindly pointed out to me by J-P.Serre. We may assume $S=0$ by translation, and then the maximal value problem will be the same whether we impose $S=0$ or do not fix $S$ (see [Sc] $\S 2$ ).

Now let us take $n=p$ and $\left\{x_{i}\right\}$ to be the conjugates of $\eta_{p}$. Then $S=0, T=p-1$, hence $a=0, b=1$. Our Corollary follows. Comparison of exponents of $p$ shows that the two sides of (2.5.3) can never be equal. 


\subsection{Concluding remarks}

This paper consists mostly of inequalities, functional and numerical, under the Generalized Riemann Hypothesis in the number field case. Computational data, including graphical ones related to $\Phi_{K}(x), \log x, \gamma_{K}$ and the upper and the lower bounds given by Proposition 2, impressively fit with the conditional results.

However, a more interesting problem related to $\gamma_{K}$ is its total behavior when we consider a natural family of $K$, for example, a family of curves over $\mathbb{F}_{p}$ arising from a two dimensional scheme over $\mathbb{Z}$ or $\mathbb{F}_{p}$. We hope to be able to report on this, too, in the near future.

[Acknowledgments] I wish to express my deep gratitude to Professor M.A.Tsfasman for valuable comments related to the NF-analogue of Theorem 2 and for providing a related text to this Volume, to Professor J-P.Serre for helpful information, to Dr.M.Shimura for all his technical assistance including those related to numerical computations, and to the referee for valuable suggestions for the improvement of presentation.

\section{References}

[D-V] V.G.Drinfeld, S.G.Vladut, On the number of points of algebraic curves, (Russian), Functional Analysis, 17(1983), 68-69.

[E-] N.Elkies, E.Howe, A.Kresch, B.Poonen, J.Wetherell, M.Zieve, Curves of every genus with many points, II: Asymptotically good families, Duke math.J. 122 (2004), 399422 .

[G] K.Gyo"ry, Discriminant form and index form equations, in "Algebraic Number Theory and Diophantine Analysis", F.Halter-Koch and R.F.Tichy (eds), de Gruyter (2000),191-214.

[G-S] A.Granville, H.M.Stark, ABC implies no "Siegel zeros" for L-functions of characters with negative discriminant, Invent. math. 139 (2000), 509-523.

[H] E.Hecke, Über die Kroneckersche Grenzformel für reelle Quadratische Körper und die Klassenzahl relativ-abelscher Körper (1917); Mathematische Werke 10; 198-207.

[HIKW] Y.Hashimoto, Y.Iijima, N.Kurokawa, M.Wakayama, Euler's constants for the Selberg and the Dedekind zeta functions, preprint 2002, to appear in Bull. Belg. Math. Soc. Simon Stevin.

$\left[\mathrm{I}_{1}\right]$ Y.Ihara, The congruence monodromy problems, J.Math.Soc.Japan,20(1968),107-121. 
$\left[\mathrm{I}_{2}\right]$ Y.Ihara, Shimura curves over finite fields and their rational points, Contemp.Math. 245 (1999), 15-23.

[L-O] J.C.Lagarias, A.M.Odlyzko, Effective versions of the Chebotarev density theorem, in Alg.N.Fields, (A.Fröhlich, Ed.) Proc. of the 1975 Durham Symposium, Academic Press, London \& New York, (1977), 409-464.

[La] S.Lang, Algebraic Number Theory, Addison-Wesley (1970).

[Le] H.W.Lenstra,Jr., Miller's primality test, Inform. Proc. Letters 8 (1979), 86-88.

[Li] J.E.Littlewood, On the class-number of the corpus $\mathrm{P}(\sqrt{-k})$, Proc.London Math.Soc.,Ser.2, Vol 27 (1928); Collected Papers II 1928,3. 920-934.

[Sc] I.Schur, Über die Verteilung der Wurzeln bei gewissen algebraischen Gleichungen mit ganzzahligen Koeffizienten, Math. Zeitschr. 1 (1918),377-402 (Gesamm. Abh. II,213-238).

[St] H.M.Stark, Some effective cases of the Brauer-Siegel Theorem, Invent. math. 23 (1974), 135-152.

$\left[\mathrm{Ts}_{1}\right]$ M.A.Tsfasman, Some remarks on the asymptotic number of points, in Springer Lect.Notes Math., 1518(1992),178-192.

$\left[\mathrm{Ts}_{2}\right]$ M.A.Tsfasman, Asymptotic behaviour of the Euler-Kronecker constant, in this Volume.

[T-V] M.A.Tsfasman, S.G.Vladut, Infinite global fields and the generalized Brauer-Siegel theorem, Moscow Math.J. 2(2002), 329-402.

[TVZ] M.A.Tsfasman, S.G.Vladut, Th.Zink, Modular curves, Shimura curves, and Goppa codes better than the Varshamov-Gilbert bound, Math.Nachr. 109 (1982), 21-28.

[W $\mathrm{W}_{1}$ ] A.Weil, [1952b] Sur les "formules explicites" de la théorie des nombres premiers, Comm. Sém. Math. Lund (1952), 252-265. (Collected Works, Vol 2, p48-62).

$\left[\mathrm{W}_{2}\right]$ A.Weil, [1972] Sur les formules explicites de la théorie des nombres (Collected Works, Vol 3, p249-264).

Department of Mathematics, Graduate School of Science and Engineering Chuo University, Kasuga 1-13-27, Bunkyo-ku, Tokyo 112-8551, Japan (email: ihara@math.chuo-u.ac.jp, ihara@kurims.kyoto-u.ac.jp) 